\title{
Modeling a deep transfer learning framework for the classification of COVID-19 radiology dataset
}

\author{
Michael Adebisi Fayemiwo ${ }^{1}$, Toluwase Ayobami Olowookere ${ }^{1}$, Samson Afolabi Arekete ${ }^{1}$, Adewale Opeoluwa \\ Ogunde $^{\text {Corresp., } 1}$, Mba Obasi Odim ${ }^{1}$, Bosede Oyenike Oguntunde ${ }^{1}$, Oluwabunmi Omobolanle Olaniyan ${ }^{1}$, Theresa \\ Omolayo Ojewumi ${ }^{1}$, Idowu Sunday Oyetade ${ }^{1}$, Ademola Adegoke Aremu ${ }^{2}$, Aderonke Anthonia Kayode \\ 1 Department of Computer Science, Redeemer's University, Ede, Osun, Nigeria \\ 2 Radiology Department, Ladoke Akintola University of Technology, Ogbomoso, Oyo, Nigeria \\ Corresponding Author: Adewale Opeoluwa Ogunde \\ Email address: ogundea@run.edu.ng
}

Severe Acute Respiratory Syndrome Coronavirus 2 (SARS-Coronavirus-2 or SARS-CoV-2), which came into existence in 2019 , is a viral pandemic that caused coronavirus disease 2019 (COVID-19) illness and death. Research showed that relentless efforts had been made to improve key performance indicators for detection, isolation, and early treatment. This paper used Deep Transfer Learning Model (DTL) for the classification of real-life COVID-19 dataset of chest X-ray images in both binary (COVID-19 or Normal) and threeclass (COVID-19, Viral-Pneumonia or Normal) classification scenarios. Four experiments were performed where fine-tuned VGG-16 and VGG-19 Convolutional Neural Networks (CNNs) with DTL were trained on both binary and three-class datasets that contain images of X-ray. The system was trained with X-ray images dataset for the detection of COVID-19. The fine-tuned VGG-16 and VGG-19 DTL were modelled by employing a batch size of 10 in 40 epochs, and Adam optimizer for weight updates, categorical cross-entropy loss function. The result showed that the fine-tuned VGG-16 and VGG-19 models produced an accuracy of $99.23 \%$ and $98.00 \%$, respectively, in the binary task. In contrast, in the multiclass (three-class) task, the fine-tuned VGG-16 and VGG-19 DTL models produced an accuracy of $93.85 \%$ and $92.92 \%$, respectively. Moreover, the fine-tuned VGG-16 and VGG-19 models have MCC of 0.98 and 0.96 respectively in the binary classification, and 0.91 and 0.89 for multiclass classification. These results showed strong positive correlations between the models' predictions and the true labels. In the two classification tasks (binary and three-class), it was observed that the fine-tuned VGG-16 DTL model had stronger positive correlations in the MCC metric than the fine-tuned VGG-19 DTL model. The VGG-16 DTL model has a Kappa value of 0.98 as against 0.96 for the VGG-19 DTL model in the binary classification task, while in the three-class classification problem, the VGG-16 DTL model has a Kappa value of 0.91 as against 0.89 for the VGG-19 DTL model. 
discovered that the VGG-16 based DTL model classified COVID-19 better than the VGG-19 based DTL model. Using the best performing fine-tuned VGG-16 DTL model, tests were carried out on 470 unlabeled images dataset, which was not used in the model training and validation processes. The test accuracy obtained for the model was $98 \%$. The proposed models provided accurate diagnostics for both the binary and multiclass classifications, outperforming other existing models in the literature in terms of accuracy, as shown in this work. 
1 Modeling a Deep Transfer Learning Framework for the 2 Classification of COVID-19 Radiology Dataset

3

4

5

6

\section{7}

Michael Adebisi Fayemiwo ${ }^{1}$, Toluwase Ayobami Olowookere', Samson Afolabi Arekete ${ }^{1}$, Adewale Opeoluwa Ogunde ${ }^{1}$, Mba Obasi Odim ${ }^{1}$, Bosede Oyenike Oguntunde ${ }^{1}$, Oluwabunmi Omobolanle Olaniyan ${ }^{1}$, Theresa Omolayo Ojewumi ${ }^{1}$, Idowu Sunday Oyetade ${ }^{1}$, Ademola Adegoke $\mathrm{Aremu}^{2}$, Aderonke Anthonia Kayode ${ }^{1}$

${ }^{1}$ Department of Computer Science, Redeemer's University, Ede, Osun State, Nigeria

${ }^{2}$ Radiology Department, Ladoke Akintola University of Technology, Ogbomoso. Oyo State. Nigeria

Corresponding Author:

Adewale Opeoluwa Ogunde ${ }^{1}$

Akoda, Ede, Osun State, 232103, Nigeria

Email address: ogundea@run.edu.ng

\section{Abstract}

Severe Acute Respiratory Syndrome Coronavirus 2 (SARS-Coronavirus-2 or SARS-CoV-2), which came into existence in 2019, is a viral pandemic that caused coronavirus disease 2019 (COVID-19) illness and death. Research showed that relentless efforts had been made to improve key performance indicators for detection, isolation, and early treatment. This paper used Deep Transfer Learning Model (DTL) for the classification of real-life COVID-19 dataset of chest X-ray images in both binary (COVID-19 or Normal) and three-class (COVID-19, ViralPneumonia or Normal) classification scenarios. Four experiments were performed where finetuned VGG-16 and VGG-19 Convolutional Neural Networks (CNNs) with DTL were trained on both binary and three-class datasets that contain images of X-ray. The system was trained with X-ray images dataset for the detection of COVID-19. The fine-tuned VGG-16 and VGG-19 DTL were modelled by employing a batch size of 10 in 40 epochs, and Adam optimizer for weight updates, categorical cross-entropy loss function. The result showed that the fine-tuned VGG-16 and VGG-19 models produced an accuracy of $99.23 \%$ and $98.00 \%$, respectively, in the binary task. In contrast, in the multiclass (three-class) task, the fine-tuned VGG-16 and VGG-19 DTL models produced an accuracy of $93.85 \%$ and $92.92 \%$, respectively. Moreover, the finetuned VGG-16 and VGG-19 models have MCC of 0.98 and 0.96 respectively in the binary classification, and 0.91 and 0.89 for multiclass classification. These results showed strong positive correlations between the models' predictions and the true labels. In the two classification tasks (binary and three-class), it was observed that the fine-tuned VGG-16 DTL model had stronger positive correlations in the MCC metric than the fine-tuned VGG-19 DTL model. The 
40 VGG-16 DTL model has a Kappa value of 0.98 as against 0.96 for the VGG-19 DTL model in 41 the binary classification task, while in the three-class classification problem, the VGG-16 DTL 42 model has a Kappa value of 0.91 as against 0.89 for the VGG-19 DTL model. This result is in 43 agreement with the trend observed in the MCC metric. Hence, it was discovered that the VGG4416 based DTL model classified COVID-19 better than the VGG-19 based DTL model. Using the 45 best performing fine-tuned VGG-16 DTL model, tests were carried out on 470 unlabeled images 46 dataset, which was not used in the model training and validation processes. The test accuracy 47 obtained for the model was $98 \%$. The proposed models provided accurate diagnostics for both 48 the binary and multiclass classifications, outperforming other existing models in the literature in 49 terms of accuracy, as shown in this work.

50

Keywords: Convolutional neural networks, Coronavirus, COVID-19 test results, Deep transfer learning, Machine learning, VGG-16, VGG-19

53

54

55

56

57

58

59

60

61

62

63

64

65

66

67

68

69

70

71

72

73

74

75

76

77

78

79

\section{Introduction}

Viral pandemics are usually a serious threat to the world, and Coronavirus disease 2019 (COVID-19) is not an exception. According to a COVID-19 report of the World Health Organization (WHO), coronaviruses are from a large family of viruses that cause illness in animals or humans (WHO, 2020). Numerous coronaviruses have been reported as the cause of respiratory disease in humans, ranging from the common cold to more serious illnesses like Middle East Respiratory Syndrome (MERS) and Extreme Acute Respiratory Syndrome (SARS) (SARS). The newly discovered coronavirus led to the cause of the 2019-novel coronavirus (COVID-19) disease. COVID-19 was initially observed in the Wuhan province of China and has spread to all parts of the world (Nadeem, 2020). COVID-19 was recognized as a contributory virus by Chinese authorities on January 72020 . The Director-General of the WHO, on the $30^{\text {th }}$ of January 2020 reported that the epidemic constitutes a Public Health Emergency of International Concern (PHEIC), based on the recommendations made by the Emergency Committee. WHO activated the R\&D Blueprint in reaction to the occurrence to speed up diagnostics, vaccines, and therapeutics for this new coronavirus (WHO, 2020). The International Committee on Taxonomy of Viruses named the novel coronavirus as "severe acute respiratory syndrome coronavirus 2 (SARS-Coronavirus-2 or SARS-CoV-2)". Globally, as of March 10, 2021, there have been $117,332,262$ confirmed cases of COVID-19, including 2,605,356 deaths, reported to the World Health Organization. Also, as of March 9, 2021, a total of 268,205,245 vaccine doses have been administered (WHO, 2021). Specifically, in Nigeria, the Nigeria Centre for Disease Control (NCDC) report on March 11, 2021, showed 394 new confirmed cases recorded. To date, 159,646 cases have been confirmed, 139,983 patients have recovered and discharged, and 1,993 deaths have been recorded in 36 states and the Federal Capital Territory (NCDC, 2021).

Numerous researchers globally are putting their efforts together on collecting data and developing solutions. The persistent focus has been on advancing key performance indicators, for example, continually enhancing the speed of case detection, segregation, and early cure. The 
80

81

82

83

84

85

86

87

88

89

90

91

92

93

94

95

96

97

98

99

100

101

102

103

104

105

106

107

108

109

110

111

112

113

114

115

116

117

118

execution of these containment procedures has been sustained and enabled by the pioneering and aggressive use of cutting-edge technologies. Measures such as immediate case detection and isolation, rigorous close contact tracing and monitoring/quarantine, and direct population/community engagement have been considered in lessening COVID-19 illness and death. This work, therefore, is aimed at using Artificial Intelligence (AI), specifically machine learning, to identify those who are at risk of contracting COVID-19 to aid early diagnosis. According to (BBC, 2020), a superhuman attempt is required to ease the deaths due to the global epidemic. AI may have been overestimated - but in the case of medicine, it already has established evidence. According to Arora, Bist, Chaurasia, and Prakash (2020), the role of AI is going to be crucial for predicting the outcome based on symptoms, CT-Scan, X-ray reports, etc.

Laboratory checking of suspected cases is characterized by extended periods of testing and an exponential rise in test requests (Kobia \& Gitaka, 2020). Quick diagnostic tests with shorter turnaround times of between 10 and 30 minutes have been developed to ease the problem. However, many are presently going through clinical validation that is not in regular use (ECDC, 2020). In the process of result expectation, there is a need to continue to self-isolate. Once results are received, there is a need to remain on self-isolation until the symptoms resolve after being in seclusion for at least 14 days. If the symptoms worsen during the seclusion time or continued after 14 days, the patient has to contact the accredited healthcare providers. The Rapid Test Kits even deliver results after hours.

Pneumonia has been described as the most severe and frequent manifestation of COVID-19 infection (Huang et al. 2020); therefore, chest imaging which includes readily available and affordable chest radiograph (X-rays), remains an essential factor in the diagnosis and evaluation of COVID-19 patients (Rubin et al., 2020). However, the availability of radiologists to report the chest images is another obstacle. Therefore, there is the need to develop computer algorithms and methods to optimize screening and early detection, which is the primary purpose of this research in which deep learning, most especially Convolutional Neural Network (CNN), is deployed. Deep learning provides the chance to increase the accuracy of the early discovery by automating the primary diagnosis of medical scans (Madan, Panchal, \& Chavan, 2019). The CNN belongs to a category of a Deep Neural Networks (DNN), which comprise several layers that are hidden like convolutional layers. The convolutional layers come with the non-linear activation function, a rectified linear unit (ReLU layer), a Pooling layer, and a fully connected normalized layer. CNN divides weights in the convolutional layer, thereby decreasing the memory footprint and increasing the performance of the network (Sasikala, Bharathi \& Sowmiya, (2018). The objective of this paper is to classify a real-life COVID-19 dataset consisting of X-ray images using a novel Deep Learning Convolutional Neural Network Model. Data from chest X-rays were used because most hospitals have X-ray machines, and the COVID-19 X-ray dataset is now available on the web. The remaining parts of this paper are organized as follows; literature review, materials and methods, experimentation and results, evaluation of results , and conclusion and future works. 


\section{Literature Review}

121

122

123

124

125

126

127

128

129

130

131

132

133

134

135

136

137

138

139

140

141

142

143

144

145

146

147

148

149

150

151

152

153

154

155

156

157

158

COVID-19 is predominantly a respiratory illness, and pulmonary appearances constitute the main presentation of the disease. SARS-CoV-2 infects the respiratory system but may also affect other organs, as reported in some studies. Renal dysfunction (Chu, Tsang \& Tang, 2005; Xu, Shi \& Wang, 2020), gastrointestinal complications (Pan, Mu \& Ren, 2020), liver dysfunction (Huang, Wang \& Li, 2020), cardiac manifestations (Zhou, She, Wang \& Ma, 2020), mediastinal findings (Valette, du Cheyron \& Goursaud, 2020), neurological abnormalities, and haematological manifestations (Song \& Shin, 2020) are among the reported extrapulmonary features. Some of the clinical symptoms of COVID-19 are cough, expectoration, asthenia, dyspnoea, muscle soreness, dry throat, pharyngeal dryness and pharyngalgia, fever, poor appetite, shortness of breath, nausea, vomiting, nasal obstruction, and rhinorrhoea. A study on COVID-19 credited to WHO, stated that the disease does not exhibit distinct symptoms, and patients' symptoms can vary from fully asymptomatic to extreme pneumonia and death (WHO, 2020). Nevertheless, certain symptoms, such as dry cough, fever, dyspnea and fatigue, were confirmed to be more prevalent in COVID-19 patients. Sore throat, nasal inflammation, fever, arthralgia, chills, diarrhoea, hemoptysis, nausea, and conjunctival congestion are some of the other clinical symptoms. (Shima, Leila, Amir \& Ali, 2020). Other non-specific symptoms include loss of smell and taste, dermatologic eruptions, delirium, and a general decline in health (Recalcati, 2020). Because of the wide range of clinical indications and the growing global burden of COVID-19, it is critical to promptly scale up diagnostic ability to diagnose the virus and its risks.

Reverse transcription-polymerase chain reaction (RT-PCR) is the primary clinical instrument currently in use detect COVID-19. It uses respiratory specimens for testing (Wang et al., 2020a). RT-PCR is used as a reference method for detecting COVID-19 patients; however, the technique is expensive, manual, complicated, time-consuming, and requires specialized medical personnel. Alternatively, X-ray imaging is an easily accessible tool that can be excellent in the COVID-19 diagnosis. Chest imaging is an essential part of evaluating respiratory complications, which remain one of the most familiar presentations ranging from acute respiratory distress syndrome to respiratory failure (Huang et al. 2020). Chest imaging has been defined as an efficient screening method for detecting pneumonia, with a sensitivity of 97.5 percent for COVID-19. (Nabila et al., 2020; NHCPRC, 2020). Provided COVID-19's preference for the respiratory system, chest radiography (X-ray), CT of the thorax, and/or Ultrasound have been verified not only as case management and screening methods for COVID-19, but also as a way of reducing infection transmission through early detection in initially False-negative RT-PCR tests. (Rubin et al., 2020). Imaging tests are useful for generating clinically actionable outcomes, which can be used to determine a diagnosis or to guide management, triage, or treatment. Costs such as the risk of exposure to radiation to the patient, the risk of COVID-19 transmission to uninfected health care staff and other patients, the use of personal protective equipment (PPE), and the need 
159 for sanitation and interruption of radiology rooms in resource-constrained settings reduce the

160

161

162

163

164

165

166

167

168

169

170

171

172

173

174

175

176

177

178

179

180

181

182

183

184

185

186

187

188

189

190

191

192

193

194

195

196

197

benefit. (Kooraki, Hosseiny, Myers \& Gholamrezanezhad, 2020).

The role of imaging includes the detection of early parenchymal lung disease, disease progression, complications, and alternative diagnoses, including acute heart failure from COVID-19 myocardial injury and pulmonary thromboembolism (Driggin et al., 2020). Although $\mathrm{CT}$ is more sensitive than chest radiography, chest radiography remains the first-line imaging modality in COVID-19 patients because of its availability, affordability, reduced radiation risk, and ease of decontamination (Fatima et al., 2020). Hence, this research work utilized chest radiographs (X-ray) for identifying COVID-19 in patients. Bilateral, peripheral, lower zone prevalent ground-glass opacities are common chest radiograph observations in COVID-19 patients. (Vancheri et al., 2020). Other possible findings include normal, unilateral, or bilateral reticular alterations, consolidations, ground-glass opacities, and pleural effusion (Hamid, Mir \& Rohela, 2020).

COVID-19 is transmitted through droplets from coughing or sneezing and on close contacts with infected persons. Propagation of droplet based on infected surfaces are considered as the major means of transmitting SARS-CoV-2'. However, patients going through screening are protected and scanned through the use of treated tools (Kooraki et al., 2020). The incubation period of COVID-19 is usually about 14 days, during which it attacks the lung. Different countries recommend personal protection equipment (PPE). According to the Centers for Disease Control and Prevention, radiology personnel should use a face mask, glasses or face shield, sleeves, and an isolation garment. A surgical cap and foot covers are required in countries with more rigorous PPE guidelines, whereas a surgical mask, goggles, or face shield are recommended in countries with less strict PPE guidelines. (CDCP, 2020). COVID-19 is highly contagious, and the symptoms begin to appear 5-6 days after contracting it either from the droplet or close contact with an infected person. According to Wang, Tang, and Wei (2020), the period between the manifestation of symptom and demise ranges from 6 - 41 days, depending on the age and immune system of the patient. The period is shorter among older people (Bai et al., 2020; Hamid, Mir \& Rohela, 2020).

COVID-19 shows some unique clinical symptoms like targeting the lower airway that manifests in sore throat, sneezing, and rhinorrhoea. In addition, the chest radiographs result in some cases possess "infiltrate in the upper lobe of the lung" related to growing dyspnea with hypoxemia (Hamid et al., 2020). COVID-19 has become endemic and rapid diagnosis is imperative to identify patients and carriers for possible isolation and treatment to curb the spread of the disease. Attempts have been made to diagnose the disease, but many are slow and not accurate in that they often give false-negative and false-positive results. This section examines some of the work done in this area.

Peer] Comput. Sci. reviewing PDF | (CS-2021:02:58415:1:1:NEW 26 May 2021) 
198

199

200

201

202

203

204

205

206

207

208

209

210

211

212

213

214

215

216

217

218

219

220

221

222

223

224

225

226

227

228

229

230

231

232

233

234

235

236

237

Over comprehensive detection of each aggressive spread chain, Lokuge et al. (2020) proposed an accurate, fast, and flexible tracking approach for spotting all residual COVID-19 group transmission. Using surveillance evaluation methods, they considered efficiency and sensitivity in the classification of population transmission chains by testing primary care fever and cough patients, hospital cases, or asymptomatic community members. They also varied the number of duplications, monitoring capacities, and the prevalence of COVID-19 and non-COVID-19 fever and cough. The findings of the study revealed that testing both syndromic fever and cough primary care presentations, as well as precise and diligent case and touch monitoring and assessment, allows for proper primary direct detection and elimination of COVID-19 population transmission. Even with optimized test sensitivity, measures such as combining these approaches could allow for increased case discovery if testing capacity is minimal. The impact analysis of movement restriction as a result of emergence of COVID-19 was carried out by Hyafil and Moria (2020). The study looked at the impact of the steps put in place in Spain to combat the epidemic. The instances and the influence of the imposed restriction to movement on the multiplicative quantity of hospitalization reports were estimated. The projected figure of instances displayed a rapid rise towards total movement restriction as imposed. The primary replication rate reduced meaningfully from 5.89 (95\% CI: 5.46-7.09) before the lockdown to 0.48 (95\% CI: $0.15-1.17$ ) after the lockdown. The study found that managing a pandemic in the magnitude of COVID-19 was very intricate and required timely decisions. The significant modifications found in the infestation rate displayed that employing inclusive participation in the first phase was vital in reducing the effect of a possible transferrable threat. This paper likewise stressed the significance of dependable up-to-date epidemiological facts to precisely measure the influence of Public Health guidelines on the virus-related outburst.

Yu et al. (2020) observed the mounting evidence that suggested that there remained a hidden collection of COVID-19 asymptomatic but transferable cases and that approximating the count of disease instances without symptoms was essential in knowing the virus and curtailing its transmission; though, it was reported that it was difficult to precisely calculate the spread of the infection. A machine learning-based fine-grained simulator (MLSim) was proposed to combine many practical reasons, such as disease development during the maturation phase, cross-region population movement, unobserved patients without symptoms, preventative measures and confinement resilience, to estimate the number of asymptomatic infection, which is critical in understanding the virus and accurately containing its spread. Digital transmission mechanisms with many unspecified variables were used to simulate the relationships between the variables, which were calculated from epidemic data using machine learning approach. When MLSim learned to closely compare and contrast real-world data, it was able to simulate the instances of patients without symptoms as well. The accessible Chinese global epidemic data helped the MLSim to train better. The analysis indicated that fine-grained machine learning simulators could improve the modelling of dynamic real-world infection transmission mechanism, which can aid in the development of balanced mitigation steps. The simulator equally showed the possibility of a great amount of undiscovered disease risk, posing a major threat to containing the 
238

239

240

241

242

243

244

245

246

247

248

249

250

251

252

253

254

255

256

257

258

259

260

261

262

263

264

265

266

267

268

269

270

271

272

273

274

275

virus. COVID-19 was modelled using a composite stochastic and deterministic concept that allowed for time-varying transmission capacity and discovery chances (Romero-Severson, Hengartner, Meadors \& Ke, 2020). Iterative particle sorting was used to adapt the model to a historical data study of occurrence and casualty figures from fifty-one countries. The report confirmed the fact that the spread rate is decreasing in forty-two of the fifty-one countries surveyed. Out of the forty-two countries, thirty-four showed a big significant proof for subcritical transmission rates, though the turndown in novel cases was moderately slow in comparison to early development rates. The study concluded that attempts to reduce the occurrence of COVID-19 by social distancing were successful. They could, however, be improved and retained in various regions to prevent the disease from resurfacing. The study also proposed other approaches to manage the virus before the relaxation of social distancing efforts.

The challenges associated with the storage and security of COVID-19 patients' data were highlighted by ElDahshan, AlHabshy, and Abutaleb (2020). The authors pointed out that the variety, volume, and variability of COVID-19 patients data required storage in NoSQL database management systems (NoSQL DBMS). It was noted that available NoSQL DBMSs were fraught with security challenges that rendered them unsuitable for storing confidential patient data. Academic institutions, research centres, and enthusiasts find it difficult to select the most suitable NoSQL DBMS because there are myriads of them without standard ways of determining the best. Thus, the study presented an inventive approach to selecting and securing NoSQL DBMS for medical information. The authors outlined the five most common NoSQL database groups, as well as the most common NoSQL DBMS forms affiliated with every one of them. In addition, their research included a comparison of the various types of NoSQL DBMS. The paper provided an efficient solution to the myriads of security challenges, ranging from authorization, authentication, encryption, and auditing in storing and securing medical information utilizing a collection of web service-based functions.

Guerrero, Brito, and Cornejo (2020) used a mathematical model to depict a sneezing individual in an urban setting with a meteorological wind of medium strength. The proliferation of airborne route was demonstrated using a Lagrangian method and a wall-modeled Large Eddy Numerical simulation. The results showed that the dimensions of two kinds of droplets differ in size: larger droplets $(400-900 \mathrm{~m})$ scatter between $2-5 \mathrm{~m}$ in $2.3 \mathrm{~s}$, whereas smaller $(100-200 \mathrm{~m})$ droplets are transported in a larger, more impressive array between $8-11 \mathrm{~m}$ by the windy conditions in $14.1 \mathrm{~s}$ on average.. Knowing the ambiguity of possible infection in this way aids in the development of solutions for the possibility of adopting tougher self-care and distance policies.

To distinguish communicable acute abdomen patients suspected of COVID-19, Zhao et al. (2020) proposed a forecasting model identified as a monogram and scale. The analytical framework was built on the basis of a retrospective case study. In a training cohort, the model was formulated using LASSO regression and multivariable logistic regression method. In the training and testing cohorts, standard curve evaluated the efficiency of the monogram, receiver 
276 operating characteristic (ROC) curves, decision curve analysis (DCA), and clinical effect curves. 277 According to the monogram, a simpler testing scale and management algorithm was developed. 278 In the testing cohort, the CIAAD monogram demonstrated strong differentiation and 279 standardization, which was approved. The CIAAD monogram was clinically useful, according to 280 decision curve research. The monogram was condensed even further into the CIAAD standard. 281 The estimated Bayesian Computation method was utilized by Vasilarou, Alachiotis, Garefalaki, 282 and Beloukas (2020) to determine the parameters of a demographic scenario engaging an 283 exponential growth of the size of the COVID-19 populations and revealed that rapid exponential 284 development in population size could sustain the monitored polymorphism patterns in COVID28519 genomes.

286 Amrane et al. (2020) adopted a genetic approach using a rapid virological diagnosis on sputum 287 and nasopharyngeal samples from suspect patients. Two real-time RT-PCR systems employing a 288 289

290

291

292 primary technique probes the envelope protein (E)-encoding gene and used a synthetic RNA positive control. The subsequent system targeted the spike protein-encoding gene (forward primer, reverse primer, and probe) and used synthetic RNA positive control methods. Bai et al. (2020) proposed the use of medical technology through the internet of things (IoT) to develop an intelligent analysis and treatment assistance programme (nCapp). The conceptual cloud-based IoT platform includes the basic IoT functions as well as a graphics processing unit (GPU). To aid in deep mining and intelligent analysis, cloud computing systems were linked to existing electronic health records, image cataloguing, picture cataloguing, and interaction. Li et al. (2020) examined chest images for the analysis of COVID-19. High-resolution Computed tomography (HRCT) was implemented for analysis of the virus infection. CT scans were taken with the following parameters: $120 \mathrm{KV}$; 100-250 mAs; collimation of $5 \mathrm{~mm}$; the pitch of 1-1.5; and 512 $\mathrm{X} 512$ matrix. The images were reconstructed by high resolution and conventional algorithms. The experiments were repeated several times, running into days for each patient. The Highresolution CT objectively evaluated lung lesions giving a better understanding of the pathogenesis of the disease.

Long et al. (2020) evaluated the suitability of Computed Tomography (CT) and real-time reverse-transcriptase-polymerase Chain Reaction (rRT-PCR). A clinical experiment with life data was executed, and the results presented showed that CT examination outperformed that of rRT-PCR at $97.2 \%$ and $84.6 \%$, respectively. In Vaishya, Javaid, Khan, and Haleem (2020), seven critical AI applications for the novel COVID-19 were recognized to perform vital roles in screening, analyzing, tracking, and predicting patients. Application areas identified comprised early detection and diagnosis of the infection, treatment monitoring, individuals contact tracing, projection of cases and mortality, drugs and vaccines development, lessening healthcare workers' assignment, and deterrence of the disease by providing updated supportive information.

Zhang, Wang, Jahanshahi, Jia, and Schmitt (2020) conducted a survey that presented some proofs of mental distress and associated predictors amongst adults in the current COVID-19 
315 pandemic in Brazil. The data was composed of 638 adults from March 25 to 28, 2020, about one 316 month after the index case was confirmed in São Paulo. Female adults, who were young, more 317 trained, and practiced less, recorded higher levels of distress, with 52 percent experiencing mild 318 to - moderate distress and 18.8 percent suffering extreme distress. The study's findings also 319 revealed that a person's distance from Sao Paulo, the epicenter, had a direct connection with the

320

321

322

323

324

325

326

327

328

329

330

331

332

333

334

335

336

337

338

339

340

341

342

343

344

345

346

347

348

349

350

351

352

353 psychological distress they were experiencing. For the older population who worked the least, the "typhoon eye effect" was more potent. Adults who lived far off the worst hit geographic area and did not go to work in the week preceding the survey were the most vulnerable. The paper concluded that recognizing the predictors of suffering would allow mental health services to improve target finding and assist the more mentally defenseless adults in the crisis. Ghafari et al. (2020) assessed the challenges and indications of concern of the COVID-19 in Iran. The heterogeneous COVID-19 casualty levels around the country in fourteen university hospitals in Tehran were investigated, and it was revealed that the recorded cases on 13/03/2020 indicated just under $10 \%$ of symptomatic patients in the population in adolescents. The finding indicated that there was a major inaccurate reporting of cases in Iran. The study suggested that strict measures be implemented throughout a period of widespread underreporting in order to prevent the healthcare system from being exhausted within a month. Further studies on efficient diagnosis, detection, and vaccine of the virus are continuing for two main reasons: partly because the disease is new, and secondly because available research efforts have not been able to address the concerns effectively. Therefore, in this paper, a deep learning modeling framework for efficient identification, classification, and provision of new insights for the diagnosis of COVID19 was presented. Also, the prediction of probable patients of the novel COVID-19 using radiology scanned images of suspected patients were shown.

Zivkovic et al. (2021) proposed a hybrid comprising machine learning Adaptive Neuro-Fuzzy Inference System (ANFIS) and enhanced Bio-inspired Beetle Antennae Search (BAS) Algorithm referred to as CESBAS-ANFIS. The hybrid study was carried out with a view to improving the existing time-series prediction algorithms for forecasting COVID-19 new cases. An improved BAS algorithm was adopted to update the parameters of ANFIS. A prediction model for the virus outbreak was formulated using ANFIS trained by the improved BAS algorithm to enhance the prediction accuracy of new cases of COVID-19. CESBAS was introduced to update ANFIS parameters, thereby solving parameters' optimization problem of machine learning techniques for prediction. Cauchy mutation operator was incorporated into the original BAS called CESBAS (Cauchy Exploration Strategy BAS) to improve exploration ability and solution diversity deficiencies observed. The proposed method consists of five layers; one input layer, two hidden layers, one layer for conclusion parameters and the output layer that presented the forecasted value. The proposed model with other hybrid models was tested under the same conditions on two datasets; one dataset from WHO and the second from the "our world in data" website, and their results were compared. CESBAS-ANFIS showed superior performance compared to other hybrid techniques such as ABC-ANFIS, BAS-ANFIS, and FPA-ANFIS. 
354

355

356

357

358

359

360

361

362

363

364

365

366

367

368

369

370

371

372

373

374

375

376

377

378

379

380

381

382

383

384

385

386

387

388

389

390

391

Irfan et al. (2021) explored the contributions of hybrid deep neural networks (HDNNs), chest Xrays and computed tomography (CT) in the detection of COVID-19. The work employed X-ray imaging and CT to develop the HDNNs for predicting the early infection of COVID-19. The HDNNs were trained and tested on five thousand (5000) images collected from five different sources (public and open), comprising 57\% males and 32\% females, and 3500 infected and 1500 healthy controls within an age group of 38-55 years. The proportion of the test dataset to the training dataset was 20:80, and classification accuracy of $99 \%$ was achieved with the HDNNs on the test dataset. The results of the performed experiments showed that the new multi-model and multi-data approach achieved improved performance over the traditional machine learning models.

Elzeki, Shams, Sarhan, Abd Elfattah, and Hassanien (2021) presented a new deep learning computer-aided scheme for rapid and seamless classification of COVID-19. Consisting of three separate COVID-19 X-ray datasets, the study presented the COVID Network (CXRVN) model for assessing grayscale chest X-ray images. The scheme was implemented on three different datasets using MatLab 2019b. A comparison was made with the pre-trained models of AlexNet, GoogleNet, and ResNet using the mini-batch gradient descent and Adam optimizer to aid the learning process. Performance evaluation results of the model using F1 score, recall, sensitivity, accuracy, and precision, with generative adversarial network (GAN) data augmentation revealed that the accuracy for the two-class classification was $96.7 \%$. In comparison, that of the threeclass classification model reached $93.07 \%$. However, the authors pointed out that increased availability of datasets could improve the performance of future methodologies. In addition, it was stated that the model could be enhanced by employing computed tomography (CT-images) and studying different updated cases of the COVID-19 X-ray images.

From the reviews presented in this section, it was observed that some of the existing works reported low accuracies, used imbalanced datasets, and there was no evidence of the use of some standard evaluation metrics such as Matthew's Correlation Coefficient and Cohen's Kappa Statistics in their approaches. The aforementioned issues were addressed in this paper. In addition, the performance of the VGG-16 and VGG-19 networks, the forms of the VGGNet Architecture, in predicting COVID-19 X-ray datatsets were compared in this study.

\section{The VGGNet Architecture}

The VGGNet, proposed by Simonyan and Zisserman (2015), is a convolutional neural network that performed very well in the ImageNet Large Scale Visual Recognition Challenge (ILSVRC) in 2014. The VGG-16 and VGG-19 networks are forms of the VGGNet Architecture. The networks accept color images with size 224 x 224 and 3 channels (Red, Green, and Blue) as their input data. The images pass through convolutional layers that are stacked on top of each other, in which there is a limited reactive field of $3 \times 3$ and stride of 1 in the convolutional filter. The convolutional kernel employs row and column padding such that the resolution before the 
392

393

394

395

396

397

398

399

400

401

402

403

404

405

406

407

408

409

410

411

412

413

414

415

416

417

418

419

420

421

422

423

424

425

426

427

428

429

convolution is retained after the processing of the images. Max-pooling is then done over a max pool window of size $2 \times 2$ with a stride of 2 (Simonyan \& Zisserman, 2015).

The network of the VGG-16 CNN has 13 convolutional layers (that is, $3 \times 3$ convolutional layers in blocks that are stacked on top of one another with growing depth). Two blocks house two $3 \times 3$ convolutional layers of the same setup in a sequential arrangement, while three blocks have three $3 \times 3$ convolutional layers of the same configuration in a sequential arrangement. As such, the VGG-16 has two contiguous blocks of two convolutional layers, with each block accompanied with a max-pooling. Also, it has three continuous blocks of three convolutional layers, with each block accompanied with a max-pooling. In all, there are five max-pooling layers in the architecture. Max pooling layer handles the reduction of the volume size after each block that contains two convolutional layers and after each block that contains three convolutional layers. The informative features are obtained by these max-pooling layers that are applied at the earlier specified stages in the network. The VGG-16 further has two fully-connected layers, each with 4,096 nodes and one fully-connected layer with 1000 nodes, one node each for each of the 1000 categories of images in the ImageNet database on which the network was pre-trained, and is followed by the SoftMax classifier (Simonyan \& Zisserman, 2015), as presented in the VGG Architecture, which can be found in Frossard et al. (2016).

The network of the VGG-19 CNN has 16 convolutional layers (that is, $3 \times 3$ convolutional layers in blocks that are stacked on top of one another with growing depth). Two blocks house two $3 \times 3$ convolutional layers of the same setup in a sequential arrangement, while three blocks have four $3 \times 3$ convolutional layers of the same configuration in a sequential arrangement. In other words, the VGG-19 has two continuous blocks of two convolutional layers, with each block accompanied with a max-pooling. It also has three contiguous blocks of four convolutional layers, with each block accompanied with a max-pooling. In all, there are five max-pooling layers in the architecture. The Max-pooling layer handles the reduction of the volume size after each block that contains two convolutional layers and after each block that contains four convolutional layers. The informative features are obtained by these max-pooling layers that are applied at the earlier specified stages in the network. The VGG-19 further has two fullyconnected layers, each with 4,096 nodes and one fully-connected layer with 1000 nodes, one node each for each of the 1000 categories of images in the ImageNet database on which the network was pre-trained, and is followed by the SoftMax classifier (Simonyan \& Zisserman, 2015).

\section{Materials \& Methods}

This study focused on diagnosing the COVID-19 chest X-ray dataset using a deep learning convolutional neural network (CNN). The CNN comprises one or more convolution layers and then followed by one or more fully connected layers as obtained in a standard multilayer neural network. COVID-19 Radiology Dataset (chest X-ray) for Annotation and Collaboration was 
430 collected from the Kaggle website (a database collated by researchers from Qatar University and 431 the University of Dhaka) with collaborators from Pakistan and Malaysia, and some medical 432 doctors), and Mendeley dataset repository. The data was preprocessed, and the median filter was 433 employed to restore the image undergoing evaluation by mitigating the severity of collection 434 degradations. Manikandarajan and Sasikala (2013) mentioned some preprocessing and 435 segmentation strategies that were used. Each data point was replaced by the average value of its 436 neighbors, and itself, in the median filter. As a result, data points that differed significantly to 437 their neighbors were removed. Following the preprocessing of the image dataset, the images 438 were sectioned by using a simulated annealing algorithm. Feature extraction and classification 439 were done using CNN. The neural network-based convolutional segmentation was implemented

440

441

442

443

444

445

446

447

448

449

450

451

452

453

454

455

456

457

458

459

460

461

462

463

464

465

466

467

468

469 in Jupyter Notebook using Python programming language and the model was built using sample datasets for the system to recognize and classify the COVID-19. The model generated can be used to develop a simple web-based application that medical personnel handling COVID-19 tests could use to input new cases and quickly predict the presence of the COVID-19, with a very high level of accuracy.

\section{Dataset Description and Preprocessing}

Chest X-ray images were selected from a repository of COVID-19 positive cases' chest X-ray images, as well as regular and viral pneumonia images, which were collated by researchers from Qatar University and the University of Dhaka, along with collaborators from Pakistan and Malaysia, and some medical doctors. There are 219 COVID-19 positive images in their current release, 1341 normal images, and 1345 viral pneumonia images (Chowdhury et al., 2020). For multiple representations, the dataset of chest X-ray images for both COVID-19 and normal cases were also selected from the Mendeley dataset repository (El-Shafai, 2020), which contains 5500 Non-COVID X-ray images and 4044 COVID-19 X-ray images. This study, therefore, adopted these multisource datasets. Due to limited computing resources, in this study, 1,300 images were selected from each category for model building and validation. In other words, 1,300 images of COVID-19 positive cases, 1,300 Normal images, and 1,300 images of viral pneumonia cases, totalling 3,900 images in all. Also, a different set of 470 images (containing 70 COVID-19 images, 200 ViralPneumonia, and 200 Normal) were selected and used for testing to obtain an impartial evaluation of a final model. The dataset used in this study can be found at Fayemiwo et al. (2021a). It should be noted here that further descriptions of the datasets were not provided by the authors of the dataset's sources.

OpenCV (Bradski and Kaehler, 2008) was used for loading and preprocessing images in the dataset. Each image was loaded and preprocessed by performing a conversion to RGB channel and changing the size of the images to $224 \times 224$ pixels to be ready for the Convolutional Neural Network. Pixel intensities were then scaled to the range $[0,1]$ and converted the data and labels to NumPy array format. Labels were then encoded using a one-hot encoder while training/testing splits were created. To ensure that the model generalizes well, data augmentation was performed by setting the random image rotation to 15 degrees, random range zooming to 0.15 , random shift 
470 of width and height to 0.2 , random shear range to 0.15 , randomly flipping half of the images

471 horizontally by setting horizontal_flip = True and fill_mode to nearest. One thousand, nine

472 hundred and fifty $(1,950)$ images were allocated initially to train the model in the binary

473 classification task, resulting in 78,000 training images after augmentation. Two thousand, nine

474 hundred and twenty-five $(2,925)$ images were allocated initially for training the model in the

475 multiclass classification task, which resulted in 117,000 training images after augmentation.

\section{The Deep-Transfer Learning Model}

477 This study employed the VGG-16 and VGG-19 Convolutional Neural Networks (CNN) with 478 Deep Transfer Learning (DTL) approach for COVID-19 detection. The Deep Transfer Learning

479 (DTL) approach focused on the storage of weights that have been grown while unraveling some 480 image classification tasks and then engaging them on a related task. Several DTL networks have 481 been proposed, some of which include VGGNet (Simonyan \& Zisserman, 2015), GoogleNet 482 (Szegedy et al., 2015), ResNet (He, Zhang, Ren \& Sun, 2016), DenseNet (Huang, Liu, Van Der 483 Maaten \& Wein, 2017) and Xception (Chollet, 2017). In this paper, VGG-16 CNN and VGG-19 $484 \mathrm{CNN}$, forms of the VGGNet, were trained on the popular ImageNet images dataset. The VGG-16 485 and VGG-19 CNN were pre-trained deep neural networks on the ImageNet for computer vision 486 (image recognition) problems, having 16 weight layers and 19 weight layers, respectively. They 487 were used as pre-trained models to help learn the distinguishing features in COVID-19 X-ray 488 images with the aid of a transfer learning approach, thus, trained DTL models for the 489 identification of COVID-19 from X-ray images.

490

491

492

493

494

495

496

497

498

499

500

501

502

503

504

505

506
As shown in the workflow in Fig. 1 and Fig. 2, respectively, to train the VGG-16 based DTL model and VGG-19 based DTL model for the detection of COVID-19, the VGG-16 CNN and VGG-19 CNN was used as pre-trained models and were fine-tuned for COVID-19 detection based on the principles of transfer learning. The weights of the lower layers of the network, which train very common characteristics from the pre-trained model, were used as feature extractors for the implementation of transfer learning with fine-tuning. Therefore, the pre-trained model's lower layers weights were frozen and therefore not updated through the training process, thus not participating in the transfer-learning process. The higher layers of the pre-trained model were used for learning task-specific features from the COVID-19 images dataset. In this case, the higher layers of the pre-trained model were unfrozen, made trainable or fine-tuned in which the weights of the layers were updated. Consequently, the layers were allowed to participate in the transfer-learning process. Each of these models ends with the SoftMax layer, which produces the outputs. The weights for the VGG-16 and VGG-19 networks were pre-trained on ImageNet, and the Fully Connected (FC) layer head was removed. From there, new fully-connected layer heads were built comprising POOL $=>\mathrm{FC}=$ SOFTMAX layers and attached on top of VGG-16 and VGG-19. The Convolutional weights of VGG-16 and VGG19 were then frozen in such a way that only the FC layer head was trained. 
507 Both binary class scenario and three-class classification scenario are considered in the workflow, 508 in which the DTL model determines the class of the chest X-ray images as either "COVID-19" 509 category or "Normal" category in the binary class scenario or as either "COVID-19" category, 510 "Viral-Pneumonia" category, or "Normal" category in the three-class classification scenario.

511 The flowchart of the experimental algorithm for the deep transfer learning models based on the 512 VGG-16 and VGG-19 networks proposed in this paper is presented in Fig. 3.

513

514 Figure 1: The Architectural Workflow of the Proposed Fine-tuned VGG-16 based Deep Transfer 515 Learning Model for COVID-19 Detection

516

517 Figure 2: The Architectural Workflow of the Proposed Fine-tuned VGG-19 based Deep Transfer 518 Learning Model for COVID-19 Detection

519

520

521 Figure 3: The Flowchart of the experimental algorithm for the DTL models based on the VGG52216 and VGG-19 networks

\section{Experimentation and Results}

524 Four different experiments were performed to classify radiological X-ray images using Deep 525 Transfer Learning approaches. Two of the experiments (Experiments A and B) include each of 526 the trained two Deep Transfer Learning models (VGG-16 and VGG-19 based) on binary class X527 ray image dataset (with COVID-19 and Normal classes). The other two experiments

528 (Experiments $\mathrm{C}$ and $\mathrm{D}$ ) include each of the trained two Deep Transfer Learning models (VGG-16 529 and VGG-19 based) on a multiclass X-ray image dataset (with COVID-19, ViralPneumonia, and 530 Normal classes). For these experiments, out of a total of 3,900 images used, 2,925 images (75\%) 531 were used for training the models, while 975 images (25\%) were used for validation and to 532 perform hyper-parameter tuning. A different set of 470 images (containing 70 COVID-19 533 images, 200 ViralPneumonia, and 200 Normal) were used for testing to obtain an impartial 534 evaluation of the final model. The code for this experiment can be found at Fayemiwo et al. 535 (2021b).

536 The performances of the proposed models were obtained from the models' generated confusion 537 matrices, using standard metrics such as accuracy, specificity, precision, recall (sensitivity), F1538 Score, Matthews Correlation Coefficient and Cohen's Kappa statistics. Equations 1, 2 and 3 
539 show the formulas for computing the three key metrics used in this article, namely Accuracy,

540 Matthews Correlation Coefficient and Cohen's Kappa statistics, respectively:

$541 \quad$ Accuracy $=\frac{T P+T N}{T P+T N+F P+F N}$

$542 \quad M C C=\frac{T P+T N-F P+F N}{\sqrt{(T P+F P)(T P+F N)(T N+F P)(T N+F N)}}$

$543 \quad$ Kappa $=\frac{(T N+T P / T N+T P+F P+F N)-((T N+F P) \times(T N+F N) / T N+T P+F P+F N)}{1-((T N+F P) \times(T N+F N) / T N+T P+F P+F N)}$

544 where TP, TN, FP and FN denote True Positive, True Negative, False Positive and False

545 Negative, respectively.

\section{Experiment $A$}

547 The first experiment was performed on the binary class dataset. A DTL model based on a pre-

548 trained VGG-16 model was trained to classify the X-ray images into the two classes of COVID-

54919 or Normal; and to detect if X-ray images are simply of the class COVID-19 or Normal. The

550 VGG-16 based DTL model summary detailing the layers and parameters in each layer of the

551 model is shown in Table 1. The fine-tuned VGG-16 based DTL model consists of 14,747,650

552 total parameters, with 32,962 of them made trainable while 14,714,688 were non-trainable. The

553 VGG-16 DTL model was modelled by employing a batch size of 10 in 40 epochs, using Adam

554 optimizer specifically for updates of weights, certain cross-entropy loss function with a learning

555 rate of $1 \mathrm{e}^{\wedge}(-2)$. Performance of the proposed fine-tuned VGG-16 based DTL model was

556 evaluated on $25 \%$ of the X-ray images.

557 Table 1: The layers and layer parameters of the proposed fine-tuned VGG-16 based DTL model 558 (Binary Classification)

559

560 The output of the confusion matrix for the binary classification as obtained from the VGG-16 561 based DTL model is shown in Table 2. Figure 4 illustrates the training loss and accuracy, the 562 validation loss and accuracy graphs of the proposed fine-tuned VGG-16 based DTL model. The 563 validation accuracy, recall, specificity, precision, F1-Score, Matthews Correlation Coefficient 564 and Cohen's Kappa statistics of the proposed fine-tuned VGG-16 based DTL model were also 565 obtained.

566 Table 2: The Confusion Matrix of the Binary classification task obtained from the fine-tuned 567 VGG-16 based DTL 
569

570 Figure 4: The Training loss and accuracy with the validation loss and accuracy curves obtained

571 for the fine-tuned VGG-16 based Deep Transfer Learning Model (For Binary Classification)

572 It was observed from Fig. 4 that the validation and training losses were slightly high in the earlier

573 epochs and then almost flattened as the training occurs in subsequent epochs. The decrease in the

574 loss values at around the 40th epoch was attributed to the fact that the fine-tuned VGG-16 based

575 DTL model was exposed to all the available X-ray images time and again during each of the

576 epochs considered during training.

577 The validation accuracy obtained for the fine-tuned VGG-16 based DTL model was 99.23\%, its 578 recall was $100 \%$, while its specificity stands at $98.48 \%$. The obtained values for the precision, 579 recall, F1-Score, Matthews Correlation Coefficient and Cohen's Kappa statistics metrics for the 580 binary classification task using the VGG-16 based DTL model are given in Table 3.

581 Table 3: The Precision, Recall, F1-Score, Matthews Correlation Coefficient and Cohen's Kappa 582 statistics obtained for the classification task using the fine-tuned VGG-16 based DTL model 583 (Binary Classification)

584

\section{Experiment $B$}

586 The second experiment was performed on the binary class dataset. A DTL model based on a pre587 trained VGG-19 model was trained to classify the X-ray images into the two classes of COVID58819 or Normal; and also, to detect if X-ray images are simply of the class COVID-19 or Normal. 589 Also, the VGG-19 based DTL model summary detailing the layers and the parameters in each 590 layer of the model is shown in Table 4. The fine-tuned VGG-19 based DTL model consists of $59120,057,346$ total parameters, with 32,962 of them made trainable while 20,024,384 were non592 trainable. The VGG-19 DTL model was modelled by employing a batch size of 10 in 40 epochs, 593 using Adam optimizer for the updates of weights, categorical cross-entropy loss function with a

594 learning rate of $1 \mathrm{e}^{\wedge}(-1)$. Performance of the proposed fine-tuned VGG-19 based DTL model was 595 evaluated on $25 \%$ of the X-ray images.

596 Table 4: The layers and layer parameters of the proposed fine-tuned VGG-19 based DTL model 597 (Binary Classification)

598

599 The output of the confusion matrix for the binary classification as obtained from the fine-tuned

600 VGG-19 based DTL model are shown in Table 5. Figure 5 illustrates the training loss and 601 accuracy along with the validation loss and accuracy graphs of the proposed fine-tuned VGG-19 
602 based DTL model. The validation accuracy, recall, specificity, precision, and F1-Score of the 603 proposed fine-tuned VGG-19 based DTL model were also obtained.

604 Table 5: The Confusion Matrix of the Binary classification task obtained from the fine-tuned 605 VGG-19 based DTL

606

607 Figure 5: The Training loss and accuracy with the validation loss and accuracy curves obtained 608 for the fine-tuned VGG-19 based Deep Transfer Learning Model (For Binary Classification)

609 It was observed from Fig. 5 that the validation and training losses were slightly moderate in the 610 earlier epochs and then decreases as the training occurs in more subsequent epochs (with a sharp 611 increase at about epochs 12 and 20). The decrease in the loss values at around the 40th epoch 612 were attributed to the fact that the fine-tuned VGG-19 based DTL model was exposed to all the 613 available X-ray images repeatedly on all the epochs considered during training.

614 The validation accuracy obtained for the fine-tuned VGG-19 based DTL model was $98.00 \%$, its 615 recall was $95.95 \%$, while its specificity stands at $100 \%$. The values obtained for the precision, 616 recall, F1-Score, Matthews Correlation Coefficient and Cohen's Kappa statistics metrics for the 617 binary classification task using the VGG-19 based DTL model are given in Table 6.

618 Table 6: The Precision, Recall, F1-Score, Matthews Correlation Coefficient and Cohen's Kappa 619 statistics obtained for the classification task using the fine-tuned VGG-19 based DTL model 620 (Binary Classification)

621

\section{Experiment $C$}

623 The third experiment was performed on the multiclass (three-class) dataset, in which a DTL 624 model based on a pre-trained VGG-16 model was trained to classify the X-ray images into the 625 three classes of COVID-19, Viral Pneumonia or Normal; and to detect if X-ray images were 626 simply of the class COVID-19 or Viral Pneumonia or Normal. The VGG-16 based DTL model 627 summary, detailing the layers and parameters in each layer of the model, is shown in Table 7. 628 The fine-tuned VGG-16 based DTL model consists of 14,747,715 total parameters, with 33,027

629 of them made trainable while 14,714,688 were non-trainable. The VGG-16 DTL model was 630 modelled by employing a batch size of 10 in 40 epochs, using Adam optimizer specifically for 631 updates of weights, certain cross-entropy loss function with a learning rate of $1 \mathrm{e}^{\wedge}(-2)$, the 632 performance of the proposed fine-tuned VGG-16 based DTL model was evaluated on $25 \%$ of the 633 X-ray images. 
634 Table 7: The layers and layer parameters of the proposed fine-tuned VGG-16 based DTL model 635 (Three-class Classification)

636

637 The output of the confusion matrix for the binary classification as obtained from the VGG-16

638 based DTL model are shown in Table 8. Figure 6 illustrates the training loss and accuracy and 639 the validation loss and accuracy graphs of the proposed fine-tuned VGG-16 based DTL model.

640 The validation accuracy, recall, specificity, precision, and F1-Score of the proposed fine-tuned

641 VGG-16 based DTL model were also obtained.

642 Table 8: The Confusion Matrix of the Three-class classification task obtained from the fine643 tuned VGG-16 based DTL

644

645

646 Figure 6: The Training loss and accuracy with the validation loss and accuracy curves obtained 647 for the fine-tuned VGG-16 based Deep Transfer Learning Model (For Three-class Classification)

648 It was observed from Fig. 6 that the validation and training losses were significantly high in the 649 earlier epochs and then slowly decrease as the training occurs in more subsequent epochs. The 650 decrease in the loss values at around the 40th epoch was attributed to the fact that the fine-tuned 651 VGG-16 based DTL model was exposed to all the available X-ray images repeatedly during each 652 of the epochs considered during training.

653 The validation accuracy obtained for the fine-tuned VGG-16 based DTL model was 93.85\%, its 654 recall was $97.98 \%$, while its specificity stands at 94.69\%. The obtained values for the precision, 655 recall, F1-Score, Matthews Correlation Coefficient and Cohen's Kappa statistics metrics for the 656 three-class classification task using the VGG-16 based DTL model are given in Table 9.

657 Table 9: The Precision, Recall, F1-Score, Matthews Correlation Coefficient and Cohen's Kappa 658 statistics obtained for the classification task using the fine-tuned VGG-16 based DTL model 659 (Three-class Classification)

660

661

\section{Experiment D}

663 The fourth experiment performed on the multiclass (three-class) dataset, in which a DTL model 664 based on a pre-trained VGG-19 model was trained to classify the X-ray images into the three 
665 classes of COVID-19, ViralPneumonia or Normal; and also, to detect if X-ray images are simply 666 of the class COVID-19 or ViralPneumonia or Normal. The VGG-19 based DTL model summary 667 detailing the layers and parameters in each layer of the model is shown in Table 10. The fine668 tuned VGG-19 based DTL model consists of 20,057,411 total parameters, with 33,027 of them 669 made trainable while 20,024,384 were non-trainable. The VGG-19 DTL model was modelled by 670 employing a batch size of 10 in 40 epochs, using Adam optimizer specifically for updates of 671 weights, certain cross-entropy loss function with a learning rate of $1 \mathrm{e}^{\wedge}(-1)$, the performance of 672 the proposed fine-tuned VGG-19 based DTL model was evaluated on the $25 \%$ of the X-ray 673 images.

674 Table 10: The layers and layer parameters of the proposed fine-tuned VGG-19 based DTL 675 model (Three-class Classification)

676

677 The output of the confusion matrix for the binary classification as obtained from the VGG-19 678 based DTL model are shown in Table 11. Figure 7 illustrates the training loss and accuracy and 679 the validation loss and accuracy graphs of the proposed fine-tuned VGG-16 based DTL model. 680 The validation accuracy, recall, specificity, precision, and F1-Score of the proposed fine-tuned 681 VGG-16 based DTL model were also obtained.

682 Table 11: The Confusion Matrix of the Three-class classification task obtained from the fine683 tuned VGG-19 based DTL.

684

685 Figure 7: The Training loss and accuracy with the validation loss and accuracy curves obtained 686 for the fine-tuned VGG-19 based Deep Transfer Learning Model (For Three-class Classification)

687 It was observed from Fig. 7 that the validation and training losses were significantly high in the 688 earlier epochs and then gradually decrease as the training occurs in more subsequent epochs. 689 This decrease in the loss values at around the 40th epoch was attributed to the fact that the fine690 tuned VGG-19 based DTL model was exposed to all the available X-ray images time and again 691 during each of the epochs considered during training.

692 The validation accuracy obtained for the fine-tuned VGG-19 based DTL model was $92.92 \%$, its 693 recall was $95.95 \%$, while its specificity stands at $89.68 \%$. The obtained values for the precision, 694 recall, F1-Score, Matthews Correlation Coefficient and Cohen's Kappa statistics metrics for the 695 three-class classification task using the VGG-19 based DTL model are given in Table 12.

696 Table 12: The Precision, Recall, F1-Score, Matthews Correlation Coefficient and Cohen's 697 Kappa statistics obtained for the classification task using the fine-tuned VGG-19 based DTL 698 model (Three-class Classification) 
699

700 It was noted from the obtained confusion matrices and the computed performance evaluation

701

702

703

704

705

706

707

708

709

710

711

712

713

714

715

716

717

718

719

720

721

722

723

724

725

726

727

728

729

730

731

metrics of the binary and three-class classification tasks that the fine-tuned VGG-16 based deep transfer learning model outperformed the fine-tuned VGG-19 based deep transfer learning model in the detection of COVID-19. Based on this, some tests were carried out on unlabeled images using the developed fine-tuned VGG-16 multi-classification model. The test was carried out to obtained an impartial evaluation of the final model. Some results of the tests are shown in Fig. 8 to Fig. 10.

Being the best performing model in this study, fine-tuned VGG-16 DTL model was tested on the test dataset of 470 images. The test accuracy obtained for the model was $98 \%$. The output results of the tests as shown in Fig. 8 to Fig. 10 show how the fine-tuned VGG-16 DTL model classified and detected each of the images as either "COVID-19", "Viral Pneumonia", or "Normal." The level of confidence in the model classification is also shown. Figure 8 shows the sample images that were detected as "COVID-19" along with the model's classification confidence accuracy values. Figure 9 shows the sample images that were detected as "Viral Pneumonia" and the model's classification confidence accuracy values. In contrast, Fig. 10 shows the sample images that were detected as "Normal" along with the model's classification confidence accuracy values.

Figure 8: COVID-19 sample test results with the predicted level of confidence value

Figure 9: Viral Pneumonia sample test results with a predicted level of confidence value

Figure 10: Normal sample test results with the predicted level of confidence value

Out of the six sample images shown in Fig. 8, only one showed a lower confidence level of $76.37 \%$, while others were above 94\%. Similar results could be seen in Fig. 10 for Normal classification, where the lowest confidence level is $78.92 \%$. However, the lowest output for Viral Pneumonia is $96.21 \%$, as shown in Fig. 9. These test results showed that the developed models could generalize and adapt to new data outside the training and validation dataset. These test results are necessary to show the adaptability of the developed models when related data is considered.

\section{Evaluation of Results}

The results obtained in this work were compared with thirteen other existing approaches in the

Peer] Comput. Sci. reviewing PDF | (CS-2021:02:58415:1:1:NEW 26 May 2021) 
732 literature. Few studies conducted before this study had used twenty-five and fifty images in each

733

734

735

736

737

738

739

740

741

742

743

744

745

746

747

748

749

750

751

752

753

754

755

756

757

758

759

760

761

762

763

764

765

766

767

768 class (Sethy \& Behra, 2020; Hemdan et al., 2020; Narin et al., 2020), while nine out of the twelve approaches benchmarked used imbalanced data (Table 9). Generally, the problem in modeling imbalanced data is that it could lead to the inability of the model to generalize, or the model can be biased towards a class with a high number of data points. Hence, in this study, an equal value of data (1,300 images) was used for each category, and this is believed to have contributed to increasing accuracies of the proposed models. At the moment, creating an automated diagnostic tool for the detection of COVID-19 suffers from the drawback of limited number of cases. To ensure the generalization of the models developed in this work, data augmentation was performed by setting the random image rotation setting to 15 degrees clockwise. The proposed new models were based on fine-tuning VGG-16 and VGG-19 methods by constructing a new fully-connected layer head consisting of POOL $=>F C=$ SOFTMAX layers and append it on top of VGG-16 and VGG-19; the Convolution weights of VGG-16 and VGG-19 were then frozen, such that only the FC layer head was trained. The fine-tuned models gave better results than other models that used ordinary pre-trained VGG-16 and VGG-19 (Apostolopoulos \& Mpesiana, 2020; Khalid \& Youness, 2020). Complete results of the comparison with thirteen other existing results from the literature are presented in Table 13, with the proposed model recording the best performance accuracy. The closest performing model to the proposed model is that of the DarkCovidNet model (Tulin et al., 2020) with $98.08 \%$ accuracy, while the proposed DTL-based VGG-16 model has $99.23 \%$ accuracy, both in the binary classification task.

Table 13: Comparison of the proposed COVID-19 diagnostic methods with other deep learning methods developed using radiology images.

\section{Conclusions and Future Work}

Several researchers around the world are combining their efforts to collect data and develop solutions for the COVID-19 pandemic problem. Laboratory testing of suspected cases characterized by long waiting periods and an exponential increase in demand for tests has hitherto constituted a significant bottleneck globally. Hence, rapid diagnostic test kits are being developed, most of which are currently undergoing clinical validation and are yet to be adopted for routine use. This paper proposed a solution using the Deep Learning Convolutional Neural Network Model to classify a real-life COVID-19 dataset of chest X-ray images into threeclasses: COVID-19, Viral-Pneumonia and Normal categories. Two experiments were performed where the VGG-16 and VGG-19 Convolutional Neural Networks (CNN) with Deep Transfer Learning (DTL) was implemented in Jupyter Notebook using Python programming language. Experimental results showed that the pre-trained VGG-16 DTL model classified COVID-19 data better than the VGG-19 based DTL model. The fine-tuned VGG-16 and VGG-19 models

Peer] Comput. Sci. reviewing PDF | (CS-2021:02:58415:1:1:NEW 26 May 2021) 
769

770

771

772

773

774

775

776

777

778

779

780

781

782

783

784

785

786

787

788

789

790

791

792

793

794

795

796

\section{7}

798

799

800

801

802

803

804

805

806

807

808

809

produced classification accuracies of $99.23 \%$ and $98.00 \%$, respectively, for binary classification and $93.85 \%$ and $92.92 \%$ for multiclass classification. The proposed model, therefore, outperformed existing methods in terms of accuracy. Moreover, the fine-tuned VGG-16 and VGG-19 models have MCC of 0.98 and 0.96 respectively in the binary classification, and 0.91 and 0.89 for multiclass classification. These results showed that there are strong positive correlations between the models' predictions and the true labels. In the two classification tasks (binary and three-class), it was observed that the fine-tuned VGG-16 DTL model had stronger positive correlations in the MCC metric than the fine-tuned VGG-19 DTL model. The VGG-16 DTL model has a Kappa value of 0.98 as against 0.96 for the VGG-19 DTL model in the binary classification task, while in the three-class classification problem, the VGG-16 DTL model has a Kappa value of 0.91 as against 0.89 for the VGG-19 DTL model. This result is in agreement with the trend observed in the MCC metric. The findings of this study have a high potential of increasing the prediction accuracy for COVID-19 disease, which would be of immense benefit to the medical field and the entire human populace as it could help save many lives from untimely death.

The researchers suspect that the better performance of the VGG-16 DTL model might be attributed to the volume of data used in the experiments; that is, the depth of layers in the VGG19 architecture may not have any significant effect on the performance when the dataset is small. This suspicion would be investigated, as a future work, when more COVID-19 data is available. Finally, the COVID-19 images from Chest CT scans are not readily available, unlike X-ray images, because of their high cost. Therefore, other future works would consider using Chest CT images to develop a more sensitive diagnostic tool for detecting viral pneumonia and COVID-19 variants. Further hyper-parameter tweaking would also be done to get more accurate results.

\section{Acknowledgements}

The authors acknowledge the efforts of Dr. (Mrs.) F. O. Unuabonah in proofreading this article and increasing its readability.

\section{References}

Amrane, S., Tissot D., Doudier H., Eldin B., Hocquart C., Mailhe M., Colson M. (2020). Rapid viral diagnosis and ambulatory management of suspected COVID-19 cases presenting at the infectious diseases referral hospital in Marseille, France, - January 31 to March 1, 2020: A respiratory virus snapshot. Travel Medicine and Infectious Disease (In Press). DOI:10.1016/j.tmaid.2020.101632

Apostolopoulos, I.D., Mpesiana, T.A (2020). COVID-19: automatic detection from X-ray images utilizing transfer learning with convolutional neural networks. Physical and Engineering Sciences in Medicine, Vol 43, 635-640. https://doi.org/10.1007/s13246-02000865-4.

Arora, K., Bist, A., Chaurasia, S., \& Prakash, R. (2020). Analysis of deep learning techniques for COVID-19 detection. International Journal of Scientific Research in Engineering and Management (IJSREM), 4(4), 1-5.

Peer] Comput. Sci. reviewing PDF | (CS-2021:02:58415:1:1:NEW 26 May 2021) 
810 Bai, L., Dawei, Y., Wang, X., Tong, L., Zhu, X., Zhong, N., . . Wang. (2020). Chinese experts' 811 consensus on the Internet of Things-aided diagnosis and treatment of coronavirus disease

812

813

814

815

816

817

818

819

820

821

822

823

824

825

826

827

828

829

830

831

832

833

834

835

836

837

838

839

840

841

842

843

844

845

846

847

848

849

850

851

852

853

2019 (COVID-19). Clinical eHealth, 3, 7-15. doi:10.1016/j.ceh.2020.03.001

BBC. (2020). British Broadcasting Corporation. Retrieved from https://www.bbc.com/news/technology-52120747

Bradski, G., and Kaehler, A. (2008). Learning OpenCV: Computer vision with the OpenCV library. O'Reilly Media, Inc.

Centres for Disease Control and Prevention [CDCP], (2020). Coronavirus Disease 2019 (COVID-19) Interim Infection Prevention and Control Recommendations. https://www.cdc.gov/coronavirus/2019-ncov/infection-control/controlrecommendationshtml\#adhere. Accessed October 1, 2020.

Chollet, F. (2017). Xception: Deep Learning with Depthwise Separable Convolutions. 2017 IEEE Conference on Computer Vision and Pattern Recognition (CVPR), (pp. 18001807). Honolulu, HI.

Chowdhury, M. E., Rahman, T., Khandakar, A., Mazhar, R., Kadir, M. A., Mahbub, Z. B., . . . Reaz, M. B. (2020, March 29). Can AI help in screening Viral and COVID-19 pneumonia? Retrieved from https://arxiv.org/abs/2003.13145; https://www.kaggle.com/tawsifurrahman/covid19-radiography-database

Chu, K. H., Tsang, W. K., Tang, C. S. (2005). Acute renal impairment in coronavirus-associated severe acute respiratory syndrome. Kidney Int., 67(2), 698-705.

Driggin, E., Madhavan, M. V., Bikdeli, B., (2020). Cardiovascular Considerations for Patients, Health Care Workers, and Health Systems During the Coronavirus Disease 2019 (COVID-19) Pandemic. J. Am. Coll. Cardiol.

ECDC. (2020). ECDC: An overview of the rapid test situation for COVID-19 diagnosis in the EU/EEA. https://dx.doi.org/10.1101/2020.03.18.20038059.

ElDahshan, K. A., AlHabshy, A. A., \& Abutaleb, G. E. (2020). Data in the time of COVID-19: a general methodology to select and secure a NoSQL DBMS for medical data. PeerJ Computer Science, 6, e297.

El-Shafai, Walid; Abd El-Samie, Fathi (2020), "Extensive COVID-19 X-ray and CT Chest Images Dataset", Mendeley Data, V3, doi: 10.17632/8h65ywd2jr.3

Elzeki, O. M., Shams, M., Sarhan, S., Abd Elfattah, M., \& Hassanien, A. E. (2021). COVID-19: a new deep learning computer-aided model for classification. PeerJ Computer Science, 7 , e358.

Fatima, S., Ratnani I, Husain M, Salim Surani (2020) Radiological Findings in Patients with COVID-19. Cureus 12(4): e7651. doi:10.7759/cureus.7651.

Fayemiwo, M., Olowookere, T., Arekete, S., Ogunde, A., Odim, M., Oguntunde, B., Olaniyan, O., Ojewumi, T., Oyetade, I., \& Aremu, A. (2021a). RAIG COVID19 compiled dataset.zip (Version 3). figshare. https://doi.org/10.6084/m9.figshare.14151854.v3 ([u'https://www.kaggle.com/tawsifurrahman/covid19-radiography-database'])

Fayemiwo, M., Olowookere, T., Arekete, S., Ogunde, A., Odim, M., Oguntunde, B., Olaniyan, O., Ojewumi, T., Oyetade, I., \& Aremu, A. (2021b). RAIG: Classification of COVID-19 Radiology Dataset (Version 2). figshare. https://doi.org/10.6084/m9.figshare.14151971

Frossard, D. (2016, June 17). VGG in TensorFlow. Retrieved May 24, 2020, from https://www.cs.toronto.edu/ frossard/post/vgg16/

Peer) Comput. Sci. reviewing PDF | (CS-2021:02:58415:1:1:NEW 26 May 2021) 
854 Ghafari, M., Hejazi, B., Karshenas, A., Dascalu, S., Ferretti, L., Ledda, A., \&Katzourakis, A.

855

856

857

858

859

860

861

862

863

864

865

866

867

868

869

870

871

872

873

874

875

876

877

878

879

880

881

882

883

884

885

886

887

888

889

890

891

892

893

894

895

896 (2020). Ongoing outbreak of COVID-19 in Iran:.medRxiv preprint. doi:10.1101/2020.04.18.20070904

Guerrero, N., Brito, J. M., \& Cornejo, P. (2020). COVID-19. Transport of respiratory droplets in a microclimatologic urban scenario. medRxiv preprint. doi:10.1101/2020.04.17.20064394

Hamid, S., Mir, M. Y., \&Rohela, G. K. (2020). Novel coronavirus disease (COVID-19): A pandemic (epidemiology, pathogenesis and potential therapeutics). New Microbe and New Infect, 35. doi:10.1016/j_nmni.2020.100679

Hemdan E.E.D., Shouman M.A. and Karar M.E. (2020), COVIDX-Net: A Framework of Deep Learning Classifiers to Diagnose COVID-19 in X-ray Images, arXiv preprint arXiv:2003.11055.

Huang C, Wang Y, Li X, Ren L, Zhao J, Hu Y, Zhang L, Fan G, Xu J, Gu X, Cheng Z, Yu T, Xia J, Wei Y, Wu W, Xie X, Yin W, Li H, Liu M, Xiao Y, Gao H, Guo L, Xie J, Wang G, Jiang R, Gao Z, Jin Q, Wang J, Cao B (2020). Clinical features of patients infected with 2019 novel coronavirus in Wuhan, China. The Lancet., 395(10223), 497-506.

Huang, G., Liu, Z., Van Der Maaten, L., \& Wein, K. Q. (2017). Densely connected convolutional networks. 2017 IEEE Conference on Computer Vision and Pattern Recognition (CVPR), (pp. 2261-2269). Honolulu, HI.

Hyafil, A., \&Moriña, D. (2020). Analysis of the impact of lockdown on the evolution of COVID-19 epidemics in Spain. medRxiv preprint, 1-20. doi:10.1101/2020.04.18.20070862

Irfan, M., Iftikhar, M. A., Yasin, S., Draz, U., Ali, T., Hussain, S., . . Glowacz, A. (2021). Role of Hybrid Deep Neural Networks (HDNNs), Computed Tomography, and Chest X-rays for the Detection of COVID-19. International Journal of Environmental Research and Public Health, 18(6), 3056.

Kobia, F., \&Gitaka, J. (2020). COVID-19: Are Africa's diagnostic challenges blunting response effectiveness? AAS Open Research, 1-11.

Kooraki, S., Hosseiny, M., Myers, L., \&Gholamrezanezhad, A. (2020). Coronavirus (COVID19) Outbreak: What the Department of Radiology Should Know. J Am Coll Radiol,17(4),447-451.

Li, M., Lei, P., Zeng, B., L. Z., Yu, P., Fan, B., . . Liu, H. (2020). Coronavirus disease (COVID19): Spectrum of CT findings and temporal progression of the disease. AcadRadiol., 27(5), 603-608. doi:10.1016/j.acra.2020.03.003.

Lokuge, K., Banks, E., Davies, S., R. L., Street, T., . . G Glass, K. (2020). Exit strategies: Optimizing feasible surveillance for detection, elimination and ongoing prevention of COVID-19 community transmission. medRxiv preprint. Doi:10.1101/2020.04.19.20071217.

Long, C., Xu, H., Shen, Q., Zhang, X., Fan, B., Wang, C., .. . Li, H. (2020). Diagnosis of the coronavirus disease (COVID-19): rRT-PCR or CT? Eur J Radiol. doi:10.1016/j.ejrad.2020.108961

Madan, B., Panchal, A., \& Chavan, D. (2019). Lung cancer detection using deep learning. 2nd International Conference on Advances in Science \& Technology (ICAST-2019). 
897

898

899

900

901

902

903

904

905

906

907

908

909

910

911

912

913

914

915

916

917

918

919

920

921

922

923

924

925

926

927

928

929

930

931

932

933

934

935

936

937

938

939

940

Manikandarajan, A., \&Sasikala, S. (2013). Detection and Segmentation of Lymph Nodes for Lung Cancer Diagnosis. National Conference on System Design and Information Processing.

Nabila, C., El Kettani, C., Amal, H., Abdelkrim, B., Mohamed, M., Said, E., Nezha, D. (2020). The epidemiological and clinical profile of COVID-19 in children: Moroccan experience of the Cheikh Khalifa University Center. The Pan African Medical Journal. 35(Supp 2):57, 1-5, DOI: 10.11604/pamj.2020.35.2.23571.

Nadeem, S. (2020). Coronavirus COVID-19: Available free literature provided by various companies, Journals and Organizations around the World. Journal of Ongoing Chemical Research, 5(1), 7-13. doi:10.5281/zenodo.3722904

Narin A., Kaya C., and Pamuk Z. (2020), Automatic Detection of Coronavirus Disease (COVID19) Using X-ray Images and Deep Convolutional Neural Networks, 2020 arXiv preprint arXiv:2003.10849.

National Health Commission of People's Republic of China [NHCPRC], (2020). Diagnosis and Treatment Protocol for Novel Coronavirus Pneumonia (Trial Version 7). 2020. Accessed September 302020

Nigeria Centre for Disease Control [NCDC], (2021). Coronavirus (COVID-19) Highlights. Abuja: Nigeria Centre for Disease Control (NCDC). Retrieved March 11, 2021, from https://covid19.ncdc.gov.ng/

Pan, L., Mu, M., Ren, H. G. (2020). Clinical characteristics of COVID-19 patients with digestive symptoms in Hubei, China: a descriptive, cross-sectional, multicenter study. Am J Gastroenterol, 115(5),766-773.

Recalcati, S. (2020). Cutaneous manifestations in COVID-19: a first perspective. J Eur Acad Dermatol Venereol, 34(5), e212.

Romero-Severson, E., Hengartner, N., Meadors, G., \&Ke, R. (2020). A decline in global transmission rates of COVID-19. medRxiv preprint. doi:10.1101/2020.04.18.20070771

Rubin, G. D., Christopher, J. R., Linda, B. H., Nicola, S., Jeffrey, P. K., Suhail, R., ... Ann, N. L. (2020). The Role of Chest Imaging in Patient Management during the COVID-19 Pandemic: A Multinational Consensus Statement from the Fleischner Society. Radiology, 296(1), 172-180. doi.org/10.1148/radiol.2020201365

Sasikala, S., Bharathi, M., \&Sowmiya, B. R. (2018). Lung cancer detection and classification using deep CNN. International Journal of Innovative Technology and Exploring Engineering (IJITEE), 8(25), 259-262.

Sethy P.K. and Behera S.K. (2020), Detection of Coronavirus Disease (COVID-19) Based on Deep Features.

Shima, B., Leila, A., Amir, R. R., \& Ali, G. (2020). Extrapulmonary manifestations of COVID19: Radiologic and clinical overview. Clinical Imaging, 66, 35-41. doi:10.1016/j.clinimag.2020.05.013

Simonyan, K., \& Zisserman, A. (2015). Very Deep Convolutional for Large-Scale Image Recognition. International Conference on Learning Representations. San Diego.

Song Y., Zheng S., Li L., Zhang X., Zhang X., Huang Z., and Chong Y. (2020). Deep learning enables accurate diagnosis of novel coronavirus (COVID-19) with CT images, medRxiv.

Song, Y.G., Shin, H.-S. (2020). COVID-19, a clinical syndrome manifesting as hypersensitivity pneumonitis. Infection Chemotherapy, 52, 110-112. 
941

942

943

944

945

946

947

948

949

950

951

952

953

954

955

956

957

958

959

960

961

962

963

964

965

966

967

968

969

970

971

972

973

974

975

976

977

978

979

980

981

982

983

984

985

Vaishya, R., Javaid, M., Khan, I. H., \& Haleem, A. (2020). Artificial Intelligence (AI) applications for COVID-19 pandemic. Diabetes and Metabolic Syndrome: Clinical Research and Review, 14, 337 - 339. doi:https://doi.org/10.1016/j.dsx.2020.04.012.

Valette, X., du Cheyron, D., Goursaud, S. (2020). Mediastinal lymphadenopathy in patients with severe COVID-19. Lancet Infect Dis., pii: S1473-3099(20)30310-8.

Vancheri, S. G., Giovanni, S., Francesco, B., Alessia, M., Costanza, C., Chandra, B., Adele, V., ... Lorenzo, P. (2020). Radiographic findings in 240 patients with COVID-19 pneumonia: time-dependence after the onset of symptoms. Eur Radiol. 1-9. doi: 10.1007/s00330-020-06967-7

Vasilarou, M., A. N., Garefalaki, J., \& Beloukas, A. (2020). Population genomics insights into the recent. bioRxiv. doi:10.1101/2020.04.21.054122.

Wang L. and Wong A. (2020), COVID-Net: A Tailored Deep Convolutional Neural Network Design for Detection of COVID-19 Cases from Chest Radiography Images, 2020 arXiv preprint arXiv:2003.09871.

Wang, W., Tang, J., \& Wei, F. (2020). Updated understanding of the outbreak of 2019 novel coronavirus (2019-nCoV) in Wuhan, China. Journal of Medical Virology, 92(4), 441447. doi:10.1002/jmv.25689

Wang, W., Xu, Y., Gao, R., Lu, R., Han, K., Wu, G., \& Tan, W. (2020a). Detection of SARSCoV-2 in different types of clinical specimens. Jama, 323(18), 1843-1844.

Wang S., Kang B., Ma J., Zeng X., Xiao M., Guo J., and Xu B. (2020b)., A deep learning algorithm using CT images to screen for Corona Virus Disease (COVID-19), medRxiv.

WHO. (2020). Report of the WHO-China Joint Mission on Coronavirus Disease 2019 (COVID19). Geneva: World Health Organization.

WHO. (2021). WHO Coronavirus (COVID-19) Dashboard. Retrieved March 11, 2021, from World Health Organization: https://covid19.who.int/

Xu X., Jiang X., Ma C., Du P., Li X., and Lv S. (2020), Deep Learning System to Screen Coronavirus Disease 2019 Pneumonia, arXiv preprint arXiv:200209334.

Xu, Z., Shi, L., Wang, Y. (2020). Pathological findings of COVID-19 associated with acute respiratory distress syndrome. Lancet Respir Med., 420-422.

Yu, Y., Liu, Y., Luo, F., Tu, W., Zhan, D., Yu, G., \& Zhou, Z. (2020). COVID-19 Asymptomatic Infection Estimation. medRxiv preprint.

doi:10.1101/2020.04.19.20068072

Zhang, S. X., Wang, Y., Jahanshahi, A. A., Jia, J., \& Schmitt, V. G. (2020). First study on mental distress in Brazil during the COVID-19 crisis. medRxiv preprint. doi:doi: https://doi.org/10.1101/2020.04.18.20070896

Zhao, B., Wei, Y., Sun, W., Qin, C., Zhou, X., Wang, Z., .. .Wang, Y. (2020). Distinguish Coronavirus Disease 2019 Patients in General Surgery Emergency by CIAAD Scale: Development and Validation of a Prediction Model Based on 822 Cases in China. medRxiv preprint. doi:10.1101/2020.04.18.20071019

Zheng C., Deng X., Fu Q., Zhou Q., Feng J., Ma H., and Wang X. (2020), Deep learning-based detection for COVID-19 from chest CT using a weak label, medRxiv, https://doi.org/10.1101/2020.03.12.20027185.

Zhou, B., She, J., Wang, Y., Ma, X. (2020). The clinical characteristics of myocardial injury 1 in severe and very severe patients with 2019 novel coronavirus disease. $J$ Infect., pii: S01634453(20)30149-3.

Peer) Comput. Sci. reviewing PDF | (CS-2021:02:58415:1:1:NEW 26 May 2021) 
986 Zivkovic, M., Bacanin, N., Venkatachalam, K., Nayyar, A., Djordjevic, A., Strumberger, I., and and beetle antennae search approach. Sustainable Cities and Society, 66(102669). doi:10.1016/j.scs.2020.102669 
Figure 1

A visualization of the VGG architecture (Source: Frossard, 2016)

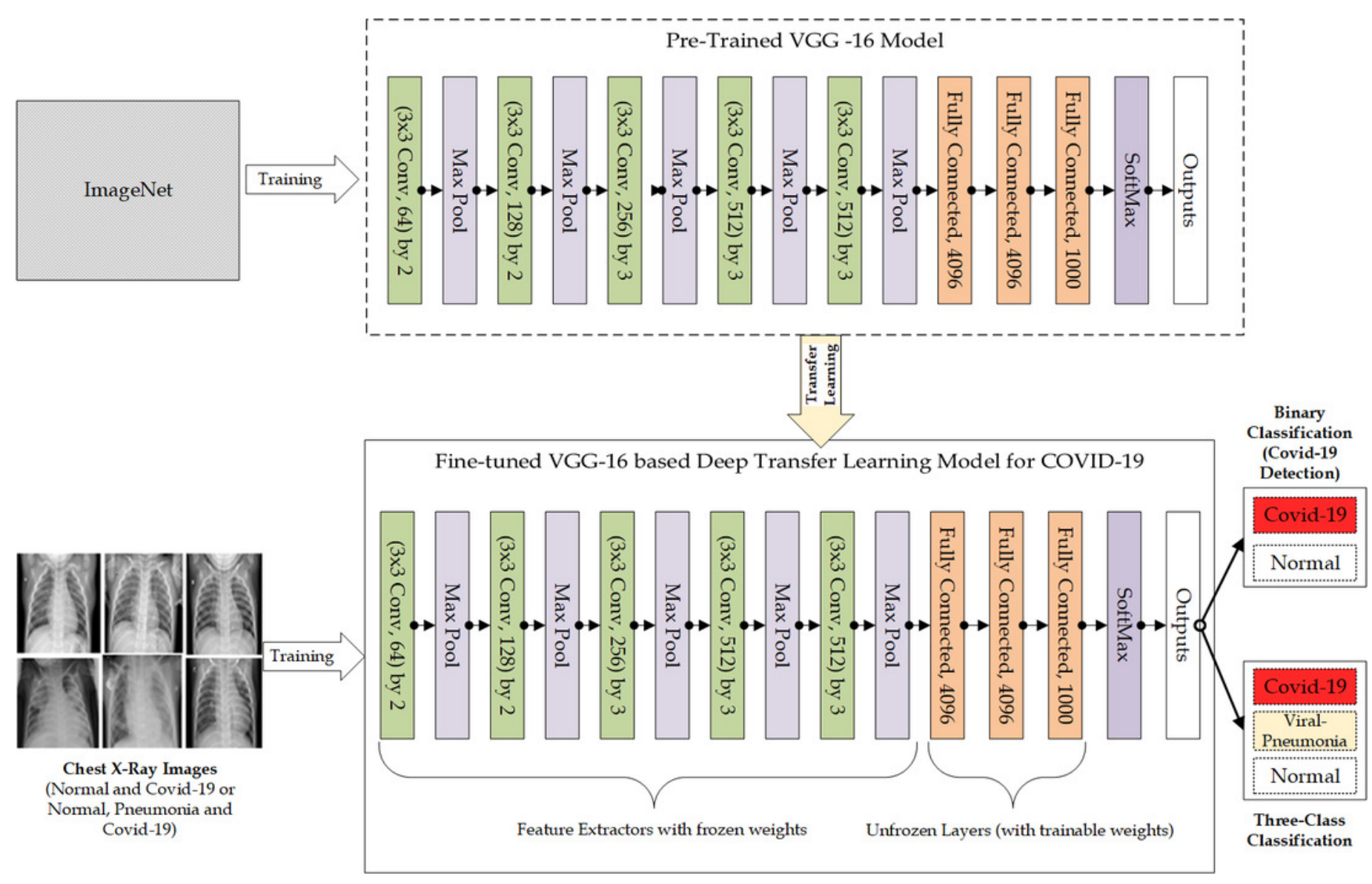


Figure 2

The Architectural Workflow of the Proposed Fine-tuned VGG-16 based Deep Transfer Learning Model for COVID-19 Detection

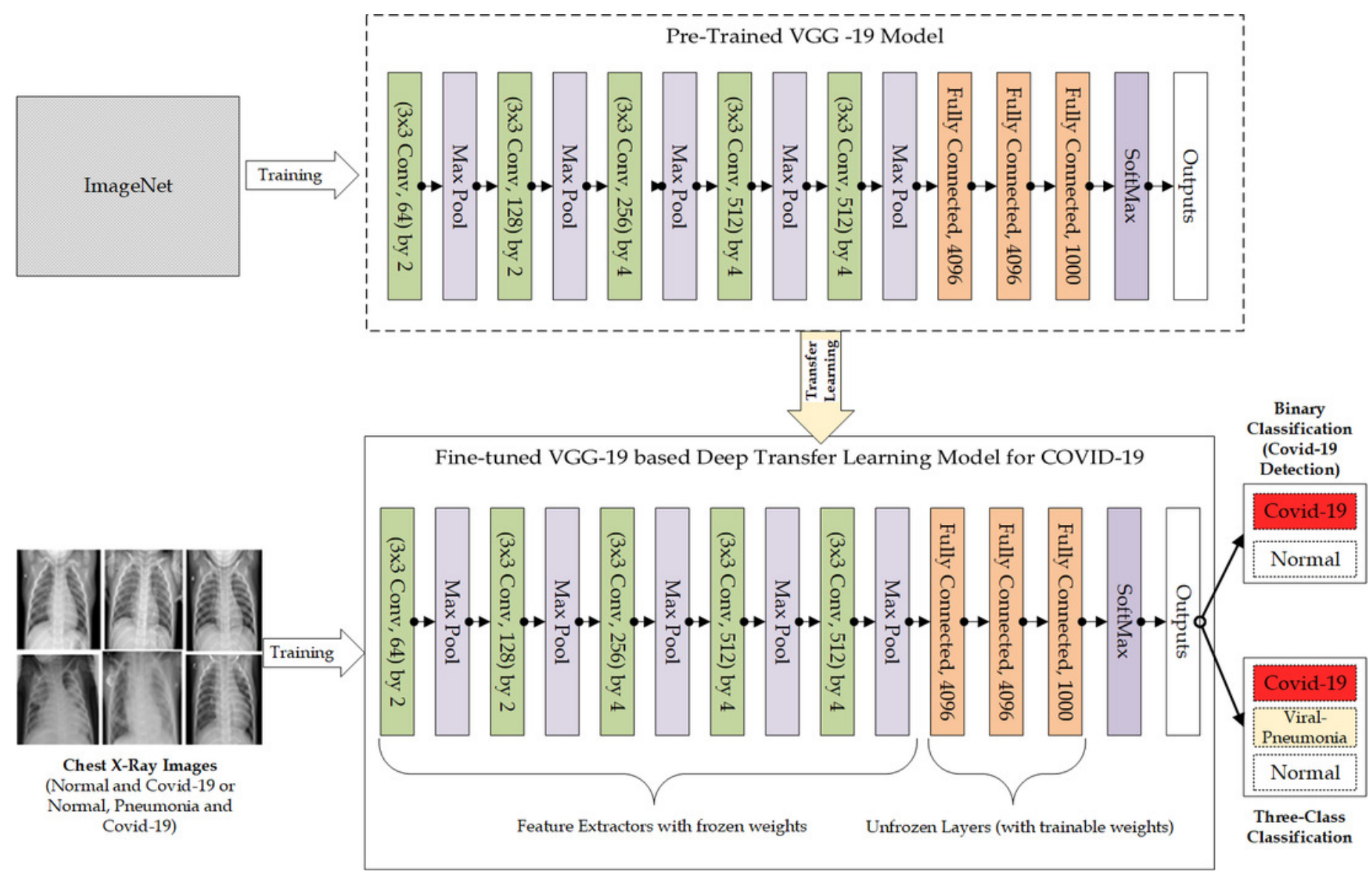


Figure 3

\section{The Architectural Workflow of the Proposed Fine-tuned VGG-19 based Deep Transfer Learning Model for COVID-19 Detection}
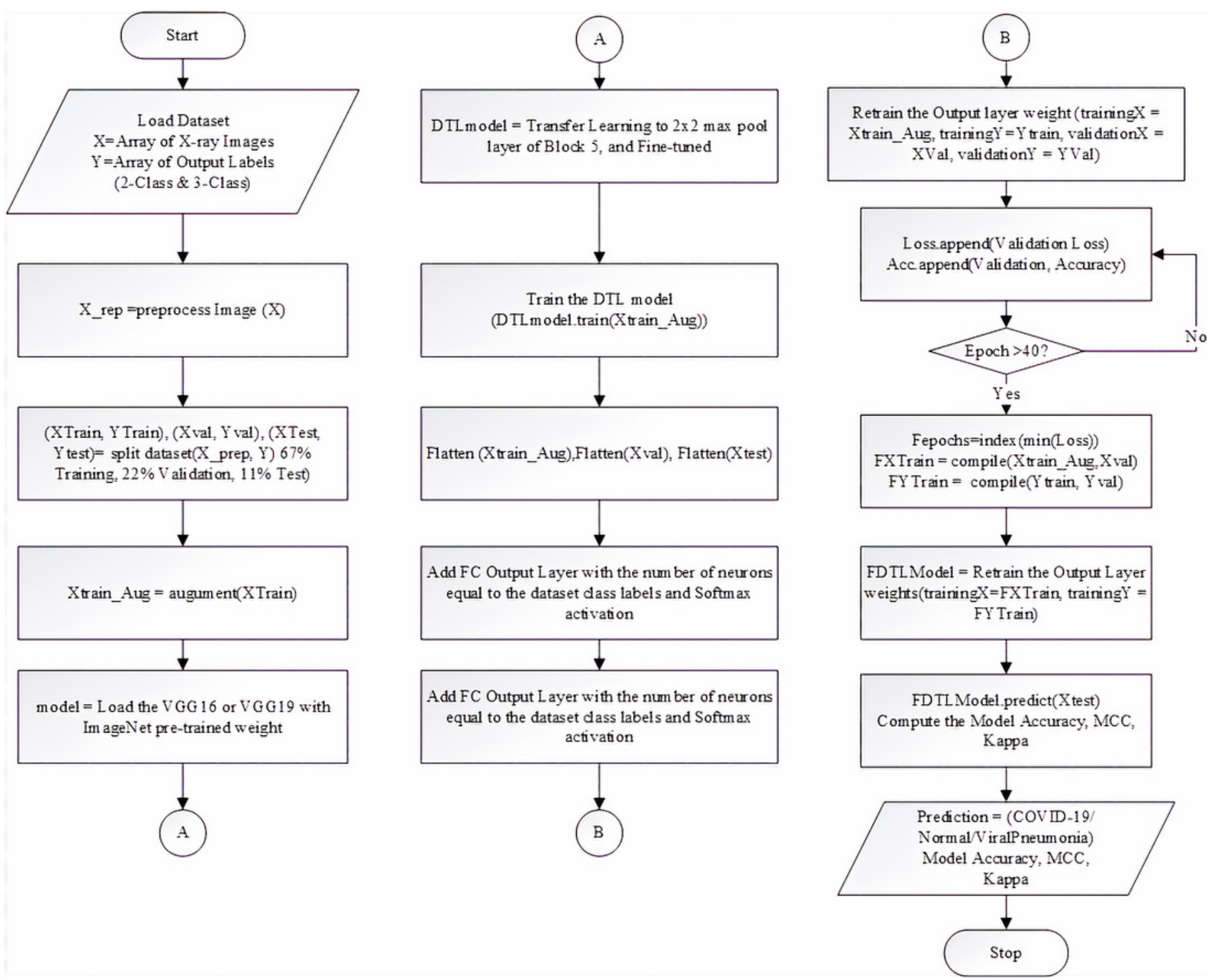
Figure 4

The Training loss and accuracy with the validation loss and accuracy curves obtained for the fine-tuned VGG-16 based Deep Transfer Learning Model (For Binary Classification)

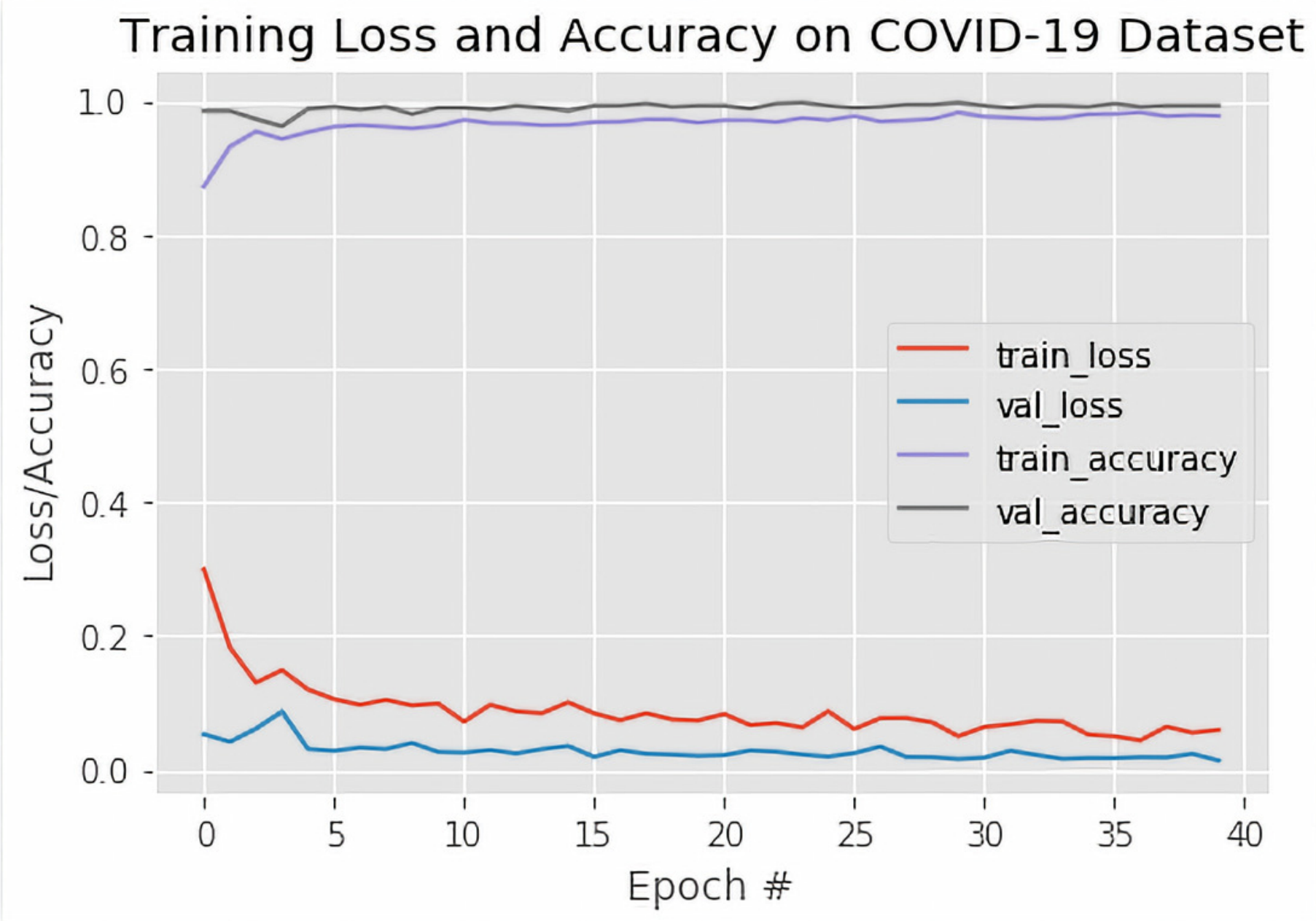


Figure 5

The Training loss and accuracy with the validation loss and accuracy curves obtained for the fine-tuned VGG-19 based Deep Transfer Learning Model (For Binary Classification)

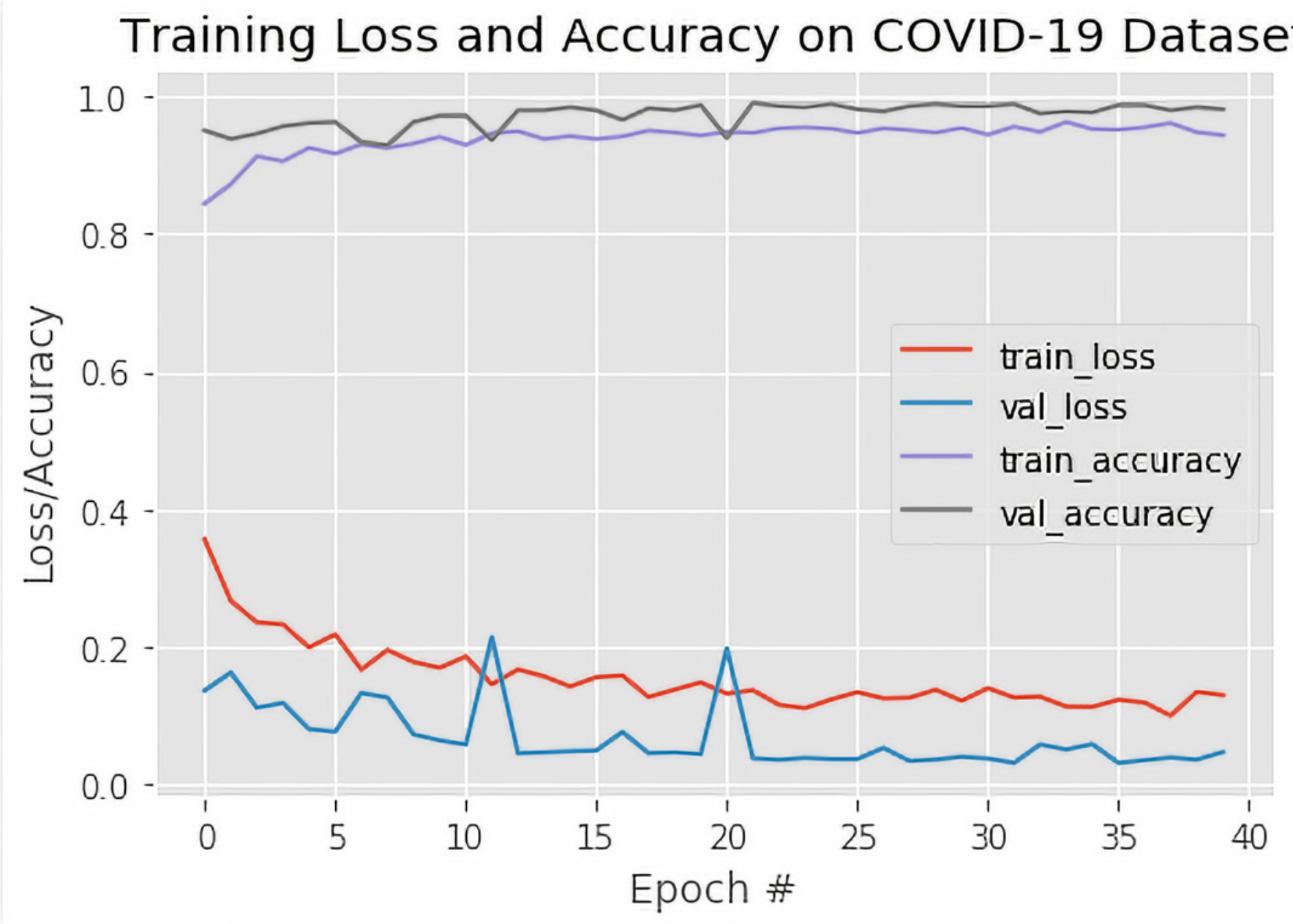


Figure 6

The Training loss and accuracy with the validation loss and accuracy curves obtained for the fine-tuned VGG-16 based Deep Transfer Learning Model (For Three-class Classification)

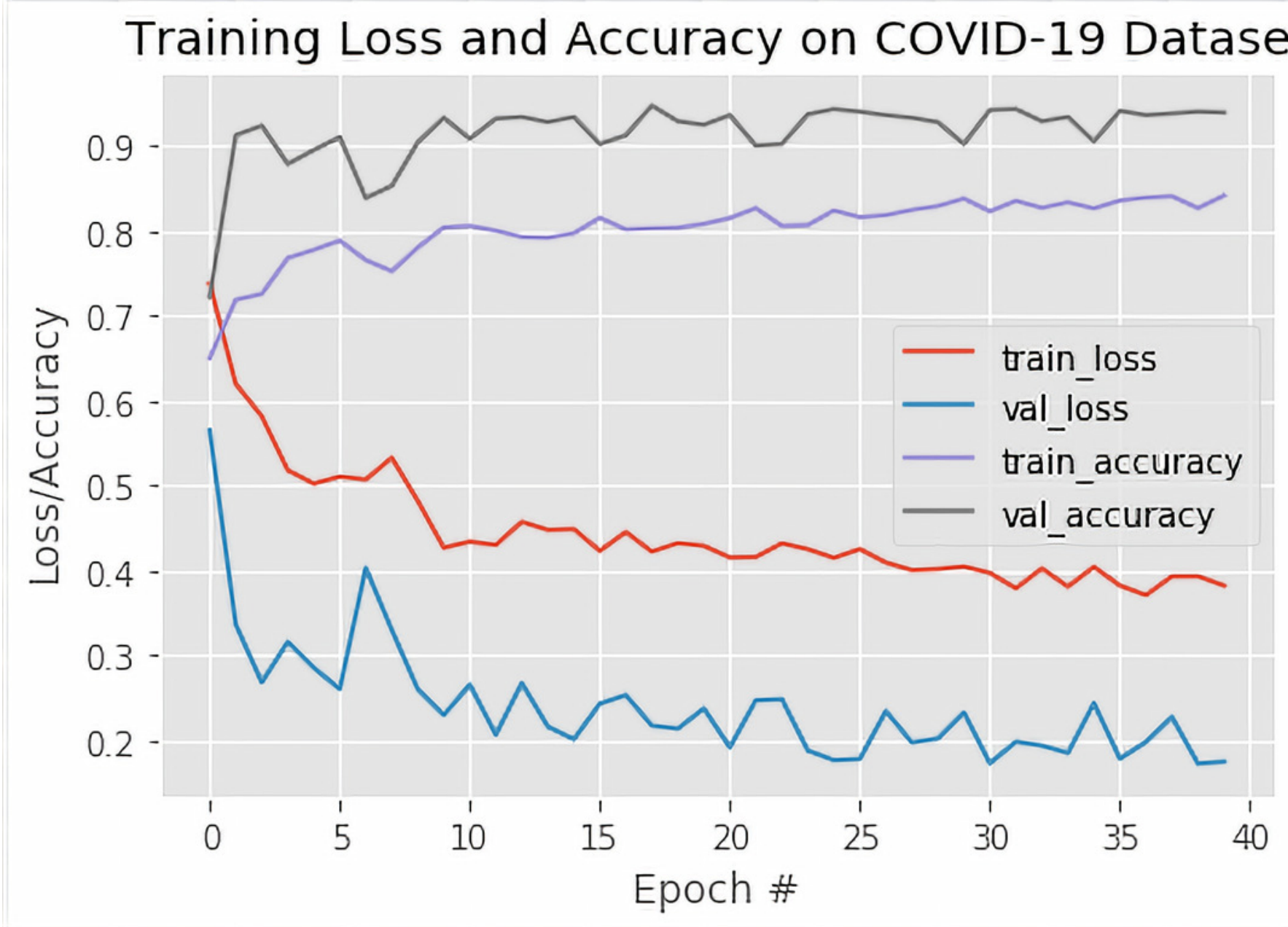


Figure 7

The Training loss and accuracy with the validation loss and accuracy curves obtained for the fine-tuned VGG-19 based Deep Transfer Learning Model (For Three-class Classification)

\section{Training Loss and Accuracy on COVID-19 Dataset}

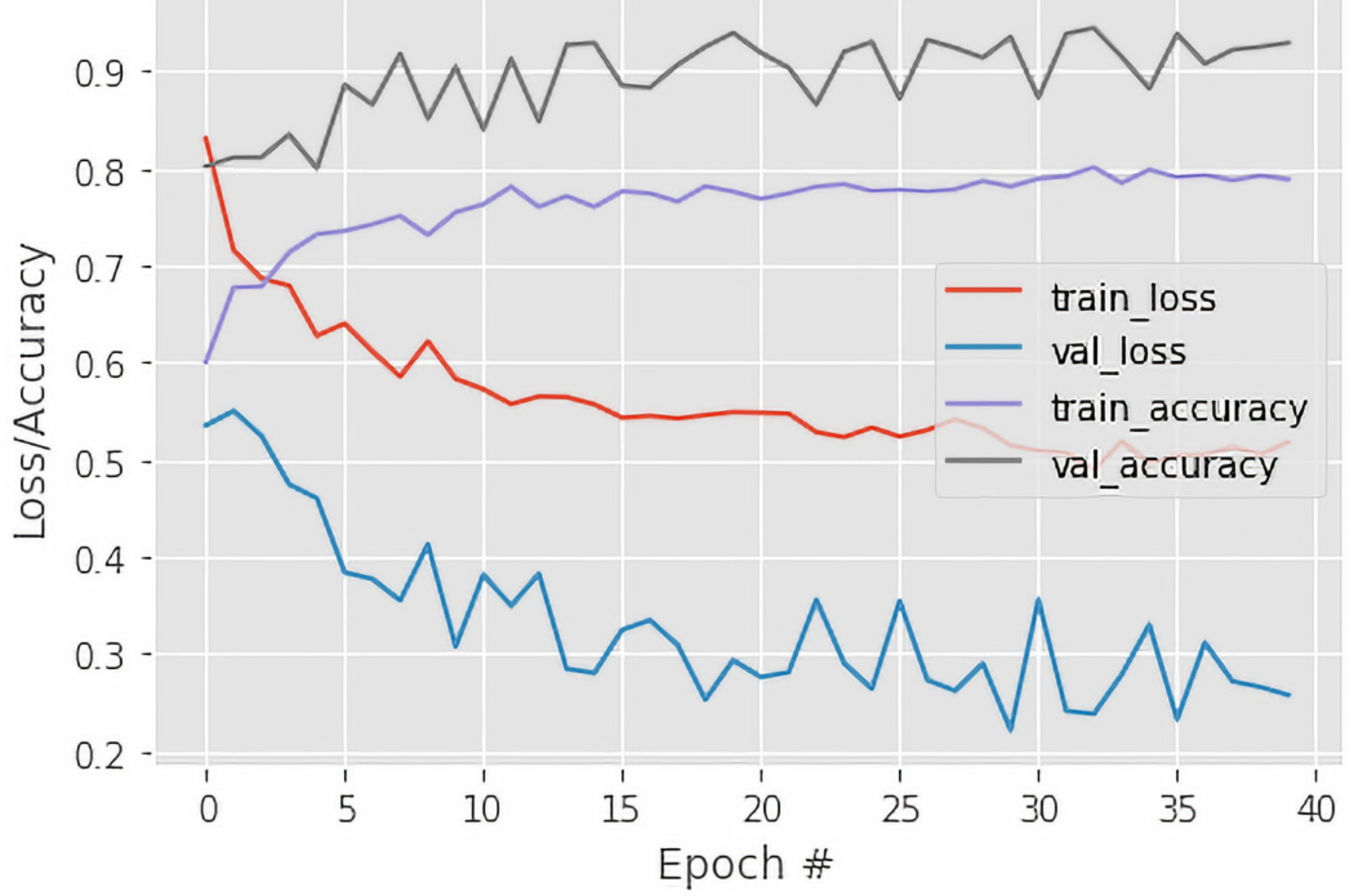


Figure 8

-19 sample test results with the predicted level of confidence value

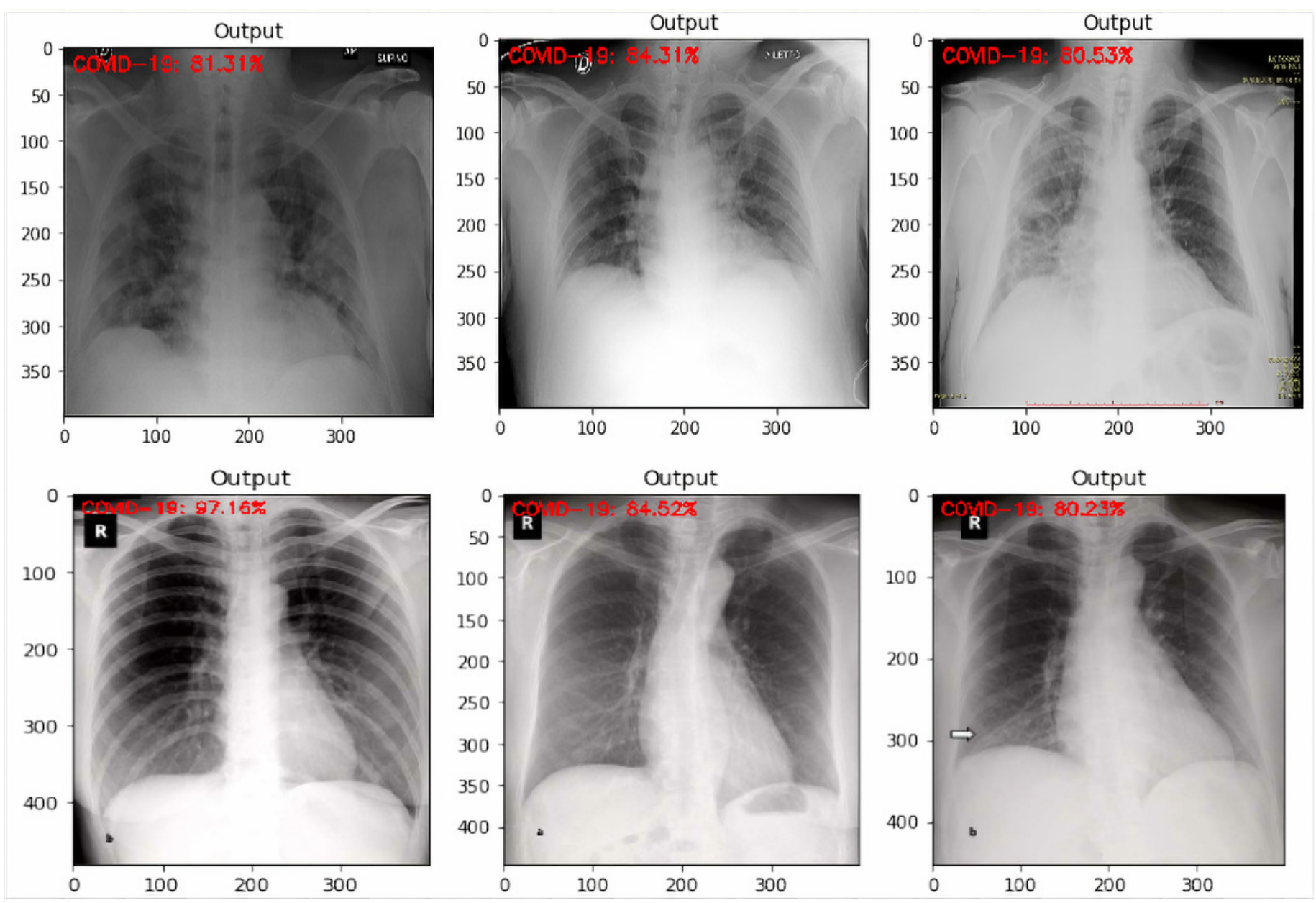


Figure 9

Viral Pneumonia sample test results with a predicted level of confidence value
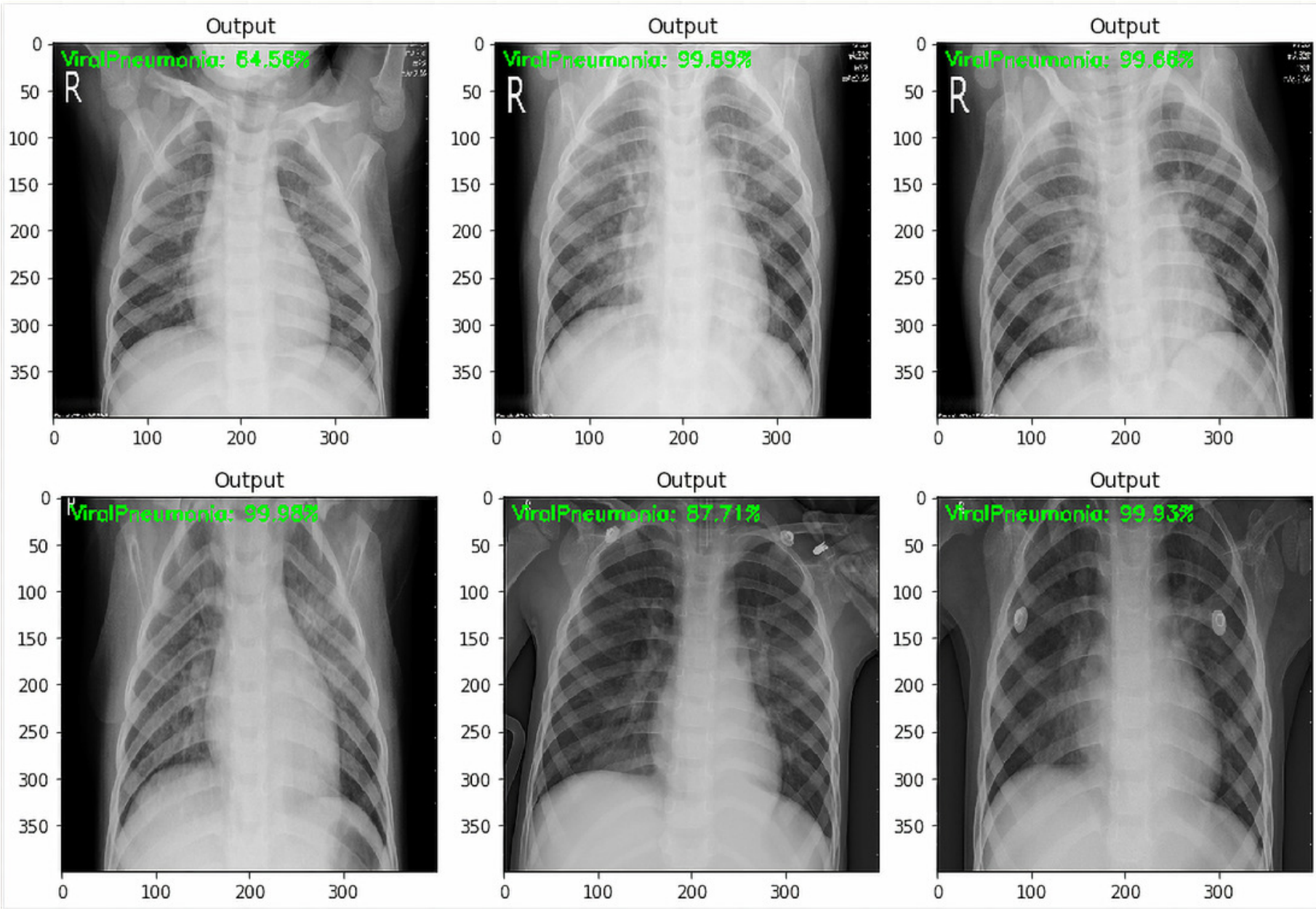
Figure 10

Normal sample test results with the predicted level of confidence value
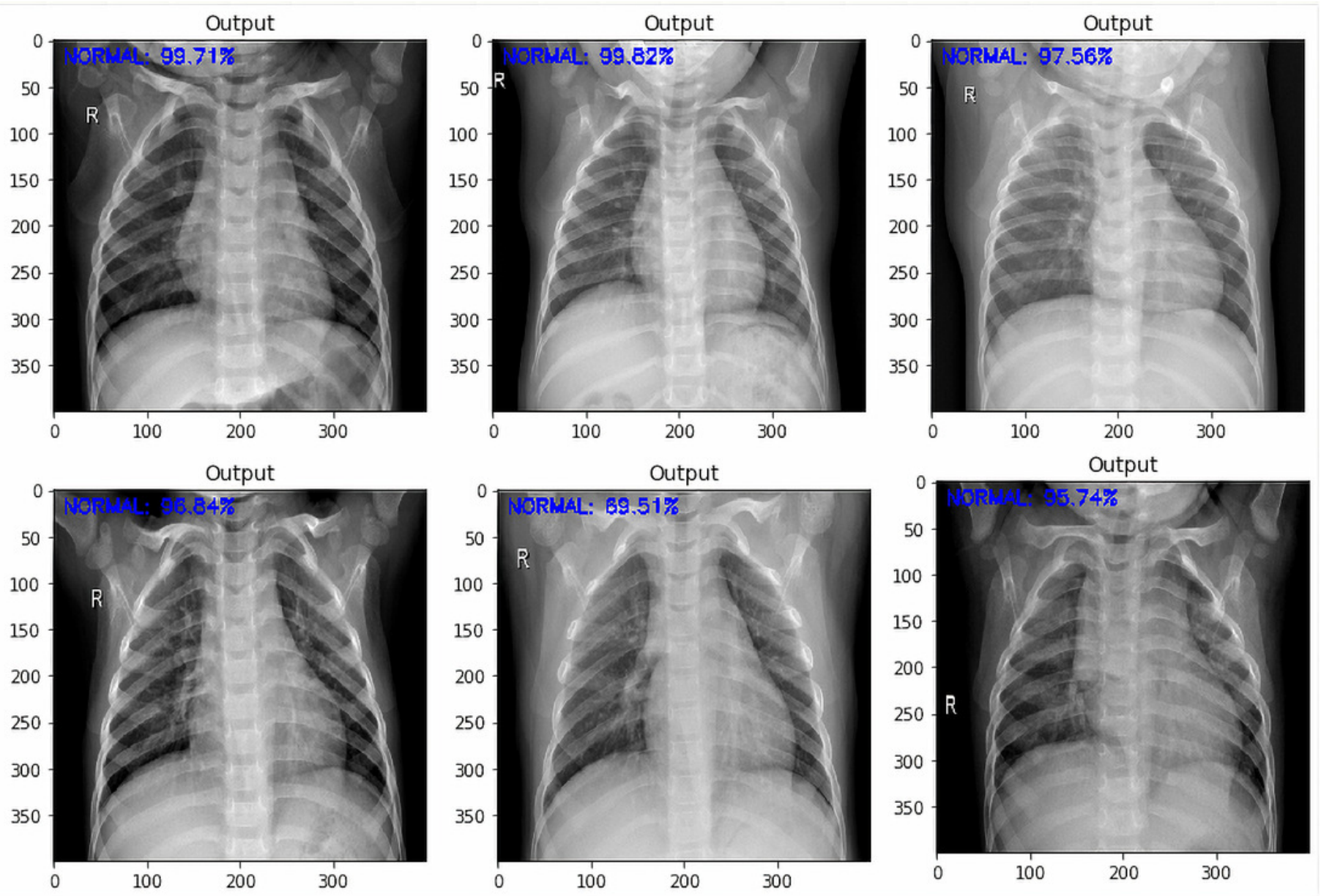


\section{Table $\mathbf{1}$ (on next page)}

The Confusion Matrix of the Binary classification task obtained from the fine-tuned VGG-16 based DTL 
1 Table 1: The layers and layer parameters of the proposed fine-tuned VGG-16 based DTL model $2 \quad$ (Binary Classification)

\begin{tabular}{|c|c|c|c|c|}
\hline & Layers & Layer's Type & $\begin{array}{l}\text { Shape of } \\
\text { Output }\end{array}$ & $\begin{array}{l}\text { Num of Trainable } \\
\text { Param }\end{array}$ \\
\hline 1 & Convolution_1 of Block_1 & $\begin{array}{l}\text { Convolution } \\
\text { 2D }\end{array}$ & {$[64,224,224]$} & 1792 \\
\hline 2 & Convolution_2 of Block_1 & $\begin{array}{l}\text { Convolution } \\
\text { 2D }\end{array}$ & {$[64,224,224]$} & 36928 \\
\hline 3 & Convolution_1 of Block_2 & $\begin{array}{l}\text { Convolution } \\
\text { 2D }\end{array}$ & {$[128,112,112]$} & 73856 \\
\hline 4 & Convolution_2of Block_2 & $\begin{array}{l}\text { Convolution } \\
\text { 2D }\end{array}$ & {$[128,112,112]$} & 147584 \\
\hline 5 & Convolution_1 of Block_3 & $\begin{array}{l}\text { Convolution } \\
\text { 2D }\end{array}$ & {$[256,56,56]$} & 295168 \\
\hline 6 & Convolution_2of Block_3 & $\begin{array}{l}\text { Convolution } \\
\text { 2D }\end{array}$ & {$[256,56,56]$} & 590080 \\
\hline 7 & Convolution_3of Block_3 & $\begin{array}{l}\text { Convolution } \\
\text { 2D }\end{array}$ & {$[256,56,56]$} & 590080 \\
\hline 8 & Convolution_1 of Block_4 & $\begin{array}{l}\text { Convolution } \\
\text { 2D }\end{array}$ & {$[512,28,28]$} & 1180160 \\
\hline 9 & Convolution_2of Block_4 & $\begin{array}{l}\text { Convolution } \\
\text { 2D }\end{array}$ & {$[512,28,28]$} & 2359808 \\
\hline 10 & Convolution_3of Block_4 & $\begin{array}{l}\text { Convolution } \\
\text { 2D }\end{array}$ & {$[512,28,28]$} & 2359808 \\
\hline 11 & Convolution_1 of Block_5 & $\begin{array}{l}\text { Convolution } \\
\text { 2D }\end{array}$ & {$[512,14,14]$} & 2359808 \\
\hline 12 & Convolution_2of Block_5 & $\begin{array}{l}\text { Convolution } \\
\text { 2D }\end{array}$ & {$[512,14,14]$} & 2359808 \\
\hline 13 & Convolution_3of Block_5 & $\begin{array}{l}\text { Convolution } \\
\text { 2D }\end{array}$ & {$[512,14,14]$} & 2359808 \\
\hline 14 & Flatten & Flatten & {$[512]$} & 0 \\
\hline
\end{tabular}




\begin{tabular}{|l|l|l|l|l|}
\hline 15 & Dense & Dense & {$[64]$} & 32832 \\
\hline 16 & Dense_1 & Dense & {$[3]$} & 130 \\
\hline
\end{tabular}




\section{Table 2 (on next page)}

The Precision, Recall and F1-Score obtained for the classification task using the finetuned VGG-16 based DTL model (Binary Classification) 
1 Table 2: The Confusion Matrix of the Binary classification task obtained from the fine-tuned 2 VGG-16 based DTL

\begin{tabular}{|l|l|l|l|}
\cline { 2 - 4 } \multicolumn{1}{c|}{} & \multicolumn{3}{c|}{ PREDICTED } \\
\hline \multirow{3}{*}{$\underbrace{*}_{2}$} & & COVID-19 & NORMAL \\
\cline { 2 - 4 } & COVID-19 & $\mathrm{TP}=321$ & $\mathrm{FN}=0$ \\
\cline { 2 - 4 } & NORMAL & $\mathrm{FP}=5$ & $\mathrm{TN}=324$ \\
\hline
\end{tabular}

3 


\section{Table 3 (on next page)}

The Confusion Matrix of the Binary classification task obtained from the fine-tuned VGG-19 based DTL 
1 Table 3: The Precision, Recall, F1-Score, MCC and Cohen's Kappa obtained for the

2 classification task using the fine-tuned VGG-16 based DTL model (Binary Classification) 3

\begin{tabular}{|l|l|l|l|c|c|}
\hline & Precision & Recall & F1-score & MCC & Cohen Kappa \\
\cline { 1 - 4 } COVID-19 & 0.98 & 1.00 & 0.99 & 0.98 & 0.98 \\
\cline { 1 - 3 } NORMAL & 1.00 & 0.98 & 0.99 & & \\
\hline
\end{tabular}

4 


\section{Table 4 (on next page)}

The Precision, Recall and F1-Score obtained for the classification task using the finetuned VGG-19 based DTL model (Binary Classification) 
1 Table 4: The layers and layer parameters of the proposed fine-tuned VGG-19 based DTL model 2 (Binary Classification)

\begin{tabular}{|c|c|c|c|c|}
\hline & Layers & Layer's Type & $\begin{array}{l}\text { Shape of } \\
\text { Output }\end{array}$ & $\begin{array}{l}\text { Num of Trainable } \\
\text { Param }\end{array}$ \\
\hline 1 & Convolution_1 of Block_1 & Convolution 2D & {$[64,224,224]$} & 1792 \\
\hline 2 & Convolution_2 of Block_1 & Convolution 2D & {$[64,224,224]$} & 36928 \\
\hline 3 & Convolution_1 of Block_2 & Convolution 2D & {$[128,112,112]$} & 73856 \\
\hline 4 & Convolution_2 of Block_2 & Convolution 2D & {$[128,112,112]$} & 147584 \\
\hline 5 & Convolution_1 of Block_3 & Convolution 2D & {$[256,56,56]$} & 295168 \\
\hline 6 & Convolution_2 of Block_3 & Convolution 2D & {$[256,56,56]$} & 590080 \\
\hline 7 & Convolution_3 of Block_3 & Convolution 2D & {$[256,56,56]$} & 590080 \\
\hline 8 & Convolution_4 of Block_3 & Convolution 2D & {$[256,56,56]$} & 590080 \\
\hline 9 & Convolution_1 of Block_4 & Convolution 2D & {$[512,28,28]$} & 1180160 \\
\hline 10 & Convolution_2 of Block_4 & Convolution 2D & {$[512,28,28]$} & 2359808 \\
\hline 11 & Convolution_3 of Block_4 & Convolution 2D & {$[512,28,28]$} & 2359808 \\
\hline 12 & Convolution_4 of Block_4 & Convolution 2D & {$[512,28,28]$} & 2359808 \\
\hline 13 & Convolution_1 of Block_5 & Convolution 2D & {$[512,14,14]$} & 2359808 \\
\hline 14 & Convolution_2 of Block_5 & Convolution 2D & {$[512,14,14]$} & 2359808 \\
\hline 15 & Convolution_3 of Block_5 & Convolution 2D & {$[512,14,14]$} & 2359808 \\
\hline 16 & Convolution_4 of Block_5 & Convolution 2D & {$[512,14,14]$} & 2359808 \\
\hline 17 & Flatten & Flatten & {$[512]$} & 0 \\
\hline 18 & Dense & Dense & [64] & 32832 \\
\hline 19 & Dense 1 & Dense & [3] & 130 \\
\hline
\end{tabular}




\section{Table 5 (on next page)}

The Confusion Matrix of the Three-class classification task obtained from the fine-tuned VGG-16 based DTL 
1 Table 5: The Confusion Matrix of the Binary classification task obtained from the fine-tuned 2 VGG-19 based DTL

3

\begin{tabular}{|c|c|c|c|}
\hline \multirow{4}{*}{ 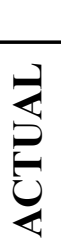 } & \multicolumn{3}{|c|}{ PREDICTED } \\
\hline & & COVID-19 & NORMAL \\
\hline & COVID-19 & $\mathrm{TP}=308$ & $\mathrm{FN}=13$ \\
\hline & NORMAL & $\mathrm{FP}=0$ & $\mathrm{TN}=329$ \\
\hline
\end{tabular}

4 


\section{Table 6(on next page)}

The Precision, Recall and F1-Score obtained for the classification task using the finetuned VGG-16 based DTL model (Three-class Classification) 
1 Table 6: The Precision, Recall, F1-Score, MCC and Cohen's Kappa obtained for the

2 classification task using the fine-tuned VGG-19 based DTL model (Binary Classification) 3

\begin{tabular}{|l|l|l|l|c|c|}
\hline & Precision & Recall & F1-score & MCC & Cohen Kappa \\
\cline { 1 - 3 } COVID-19 & 1.00 & 0.96 & 0.98 & 0.96 & 0.96 \\
\cline { 1 - 2 } NORMAL & 0.96 & 1.00 & 0.98 & & \\
\hline
\end{tabular}




\section{Table 7 (on next page)}

The Confusion Matrix of the Three-class classification task obtained from the fine-tuned VGG-19 based DTL. 
1 Table 7: The layers and layer parameters of the proposed fine-tuned VGG-16 based DTL model 2 (Three-class Classification)

\begin{tabular}{|c|c|c|c|c|}
\hline & Layers & Layer's Type & $\begin{array}{l}\text { Shape of } \\
\text { Output }\end{array}$ & $\begin{array}{l}\text { Num of Trainable } \\
\text { Param }\end{array}$ \\
\hline 1 & Convolution_1 of Block_1 & $\begin{array}{l}\text { Convolution } \\
\text { 2D }\end{array}$ & {$[64,224,224]$} & 1792 \\
\hline 2 & Convolution_2 of Block_1 & $\begin{array}{l}\text { Convolution } \\
\text { 2D }\end{array}$ & {$[64,224,224]$} & 36928 \\
\hline 3 & Convolution_1 of Block_2 & $\begin{array}{l}\text { Convolution } \\
\text { 2D }\end{array}$ & {$[128,112,112]$} & 73856 \\
\hline 4 & Convolution_2 of Block_2 & $\begin{array}{l}\text { Convolution } \\
\text { 2D }\end{array}$ & {$[128,112,112]$} & 147584 \\
\hline 5 & Convolution_1 of Block_3 & $\begin{array}{l}\text { Convolution } \\
\text { 2D }\end{array}$ & {$[256,56,56]$} & 295168 \\
\hline 6 & Convolution_2 of Block_3 & $\begin{array}{l}\text { Convolution } \\
\text { 2D }\end{array}$ & {$[256,56,56]$} & 590080 \\
\hline 7 & Convolution_3 of Block_3 & $\begin{array}{l}\text { Convolution } \\
\text { 2D }\end{array}$ & {$[256,56,56]$} & 590080 \\
\hline 8 & Convolution_1 of Block_4 & $\begin{array}{l}\text { Convolution } \\
\text { 2D }\end{array}$ & {$[512,28,28]$} & 1180160 \\
\hline 9 & Convolution_2 of Block_4 & $\begin{array}{l}\text { Convolution } \\
\text { 2D }\end{array}$ & {$[512,28,28]$} & 2359808 \\
\hline 10 & Convolution_3 of Block_4 & $\begin{array}{l}\text { Convolution } \\
\text { 2D }\end{array}$ & {$[512,28,28]$} & 2359808 \\
\hline 11 & Convolution_1 of Block_5 & $\begin{array}{l}\text { Convolution } \\
\text { 2D }\end{array}$ & {$[512,14,14]$} & 2359808 \\
\hline 12 & Convolution_2 of Block_5 & $\begin{array}{l}\text { Convolution } \\
\text { 2D }\end{array}$ & {$[512,14,14]$} & 2359808 \\
\hline 13 & Convolution_3 of Block_5 & $\begin{array}{l}\text { Convolution } \\
\text { 2D }\end{array}$ & {$[512,14,14]$} & 2359808 \\
\hline 14 & Flatten & Flatten & [512] & 0 \\
\hline
\end{tabular}




\begin{tabular}{|l|l|l|l|l|}
\hline 15 & Dense & Dense & {$[64]$} & 32832 \\
\hline 16 & Dense_1 & Dense & {$[3]$} & 195 \\
\hline
\end{tabular}

3 


\section{Table 8(on next page)}

The Precision, Recall and F1-Score obtained for the classification task using the finetuned VGG-19 based DTL model (Three-class Classification) 
1 Table 8: The Confusion Matrix of the Three-class classification task obtained from the fine2 tuned VGG-16 based DTL

3

\begin{tabular}{|c|c|c|c|c|c|}
\hline \multirow{5}{*}{$\underset{2}{2}$} & \multicolumn{5}{|c|}{ PREDICTED } \\
\hline & & COVID-19 & NORMAL & ViralPneumonia & TOTAL \\
\hline & COVID-19 & 339 & 1 & 6 & 346 \\
\hline & NORMAL & 0 & 321 & 18 & 339 \\
\hline & ViralPneumonia & 4 & 31 & 255 & 290 \\
\hline & TOTAL & 343 & 353 & 279 & 975 \\
\hline
\end{tabular}

4 


\section{Table 9 (on next page)}

Comparison of the proposed COVID-19 diagnostic methods with other deep learning methods developed using radiology images. 
1 Table 9: The Precision, Recall, F1-Score, MCC and Cohen's Kappa obtained for the

2 classification task using the fine-tuned VGG-16 based DTL model (Three-class Classification) 3

4

\begin{tabular}{|l|l|l|l|l|c|}
\hline & Precision & Recall & F1-score & MCC & Cohen Kappa \\
\cline { 1 - 3 } COVID-19 & 0.99 & 0.98 & 0.98 & & \multirow{2}{*}{0.91} \\
\cline { 1 - 3 } NORMAL & 0.91 & 0.95 & 0.93 & & \\
\cline { 1 - 3 } ViralPneumonia & 0.91 & 0.88 & 0.90 & & \\
\hline
\end{tabular}




\section{Table $\mathbf{1 0}$ (on next page)}

The layers and layer parameters of the proposed fine-tuned VGG-19 based DTL model (Three-class Classification) 
1 Table 10: The layers and layer parameters of the proposed fine-tuned VGG-19 based DTL model 2 (Three-class Classification)

\begin{tabular}{|c|c|c|c|c|}
\hline & Layers & Layer's Type & $\begin{array}{l}\text { Shape of } \\
\text { Output }\end{array}$ & $\begin{array}{l}\text { Num of Trainable } \\
\text { Param }\end{array}$ \\
\hline 1 & Convolution_1 of Block_1 & $\begin{array}{l}\text { Convolution } \\
\text { 2D }\end{array}$ & {$[64,224,224]$} & 1792 \\
\hline 2 & Convolution_2 of Block_1 & $\begin{array}{l}\text { Convolution } \\
\text { 2D }\end{array}$ & {$[64,224,224]$} & 36928 \\
\hline 3 & Convolution_1 of Block_2 & $\begin{array}{l}\text { Convolution } \\
\text { 2D }\end{array}$ & {$[128,112,112]$} & 73856 \\
\hline 4 & Convolution_2 of Block_2 & $\begin{array}{l}\text { Convolution } \\
\text { 2D }\end{array}$ & {$[128,112,112]$} & 147584 \\
\hline 5 & Convolution_1 of Block_3 & $\begin{array}{l}\text { Convolution } \\
\text { 2D }\end{array}$ & {$[256,56,56]$} & 295168 \\
\hline 6 & Convolution_2 of Block_3 & $\begin{array}{l}\text { Convolution } \\
\text { 2D }\end{array}$ & {$[256,56,56]$} & 590080 \\
\hline 7 & Convolution_3 of Block_3 & $\begin{array}{l}\text { Convolution } \\
\text { 2D }\end{array}$ & {$[256,56,56]$} & 590080 \\
\hline 8 & Convolution_1 of Block_4 & $\begin{array}{l}\text { Convolution } \\
\text { 2D }\end{array}$ & {$[512,28,28]$} & 1180160 \\
\hline 9 & Convolution_2 of Block_4 & $\begin{array}{l}\text { Convolution } \\
\text { 2D }\end{array}$ & {$[512,28,28]$} & 2359808 \\
\hline 10 & Convolution_3 of Block_4 & $\begin{array}{l}\text { Convolution } \\
\text { 2D }\end{array}$ & {$[512,28,28]$} & 2359808 \\
\hline 11 & Convolution_1 of Block_5 & $\begin{array}{l}\text { Convolution } \\
\text { 2D }\end{array}$ & {$[512,14,14]$} & 2359808 \\
\hline 12 & Convolution_2 of Block_5 & $\begin{array}{l}\text { Convolution } \\
\text { 2D }\end{array}$ & {$[512,14,14]$} & 2359808 \\
\hline 13 & Convolution_3 of Block_5 & $\begin{array}{l}\text { Convolution } \\
\text { 2D }\end{array}$ & {$[512,14,14]$} & 2359808 \\
\hline 14 & Flatten & Flatten & {$[512]$} & 0 \\
\hline
\end{tabular}




\begin{tabular}{|l|l|l|l|l|}
\hline 15 & Dense & Dense & {$[64]$} & 32832 \\
\hline 16 & Dense_1 & Dense & {$[3]$} & 195 \\
\hline
\end{tabular}

3 


\section{Table $\mathbf{1 1}$ (on next page)}

The Confusion Matrix of the Three-class classification task obtained from the fine-tuned VGG-19 based DTL 
1 Table 11: The Confusion Matrix of the Three-class classification task obtained from the fine2 tuned VGG-19 based DTL

3

\begin{tabular}{|c|c|c|c|c|c|}
\hline \multirow{5}{*}{$\underset{\psi}{己}$} & \multicolumn{5}{|c|}{ PREDICTED } \\
\hline & & COVID-19 & NORMAL & ViralPneumonia & TOTAL \\
\hline & COVID-19 & 332 & 1 & 13 & 346 \\
\hline & NORMAL & 2 & 304 & 33 & 339 \\
\hline & ViralPneumonia & 1 & 19 & 270 & 290 \\
\hline & TOTAL & 335 & 324 & 316 & 975 \\
\hline
\end{tabular}

4 


\section{Table 12 (on next page)}

The Precision, Recall, F1-Score, Matthews Correlation Coe $\square$ cient and Cohers Kappa statistics obtained for the classifiation task using the fie-tuned VGG-19 based DTL model (Three-class Classifiation) 
1 Table 12: The Precision, Recall, F1-Score, MCC and Cohen's Kappa obtained for the

2 classification task using the fine-tuned VGG-19 based DTL model (Three-class Classification) 3

\begin{tabular}{|l|l|l|l|l|c|}
\hline & Precision & Recall & F1-score & MCC & Cohen Kappa \\
\cline { 1 - 3 } COVID-19 & 0.99 & 0.96 & 0.98 & & \multirow{2}{*}{0.89} \\
\cline { 1 - 3 } & 0.94 & 0.90 & 0.92 & 0.89 \\
\cline { 1 - 3 } ViralPneumonia & 0.85 & 0.93 & 0.89 & & \\
\cline { 1 - 3 }
\end{tabular}

4 


\section{Table 13 (on next page)}

Comparison of the proposed COVID-19 diagnostic methods with other deep learning methods developed using radiology images. 
1 Table 13: Comparison of the proposed COVID-19 diagnostic methods with other deep learning

2 methods developed using radiology images.

\begin{tabular}{|c|c|c|c|c|c|}
\hline S/No & Study & $\begin{array}{l}\text { Type of } \\
\text { Images }\end{array}$ & Number of Cases & Method Used & $\begin{array}{l}\text { Accuracy } \\
(\%)\end{array}$ \\
\hline 1 & $\begin{array}{l}\frac{\text { Apostolopoulos }}{\text { and Mpesiana }} \\
\underline{(2020)}\end{array}$ & Chest X-ray & $\begin{array}{l}224 \text { COVID- } \\
19(+) \\
700 \text { Pneumonia } \\
504 \text { Healthy }\end{array}$ & VGG-19 & 93.48 \\
\hline 2 & $\frac{\text { Wang and }}{\text { Wong }(2020)}$ & Chest X-ray & $\begin{array}{l}53 \text { COVID-19(+) } \\
5526 \text { COVID-19 } \\
(-) \\
8066 \text { Healthy }\end{array}$ & COVID-Net & 92.4 \\
\hline 3 & $\begin{array}{l}\text { Sethy and Behra } \\
\underline{(2020)}\end{array}$ & Chest X-ray & $\begin{array}{l}25 \text { COVID-19(+) } \\
25 \text { COVID-19(-) }\end{array}$ & ResNet50+ SVM & 95.38 \\
\hline 4 & Hemdan et al. & Chest X-ray & $\begin{array}{l}25 \text { COVID-19(+) } \\
25 \text { Normal }\end{array}$ & COVIDX-Net & 90.0 \\
\hline 5 & $\frac{\text { Narin et al. }}{(2020)}$ & Chest X-ray & $\begin{array}{l}50 \text { COVID-19(+) } \\
50 \text { COVID-19(-) }\end{array}$ & $\begin{array}{l}\text { Deep CNN } \\
\text { ResNet-50 }\end{array}$ & 98.0 \\
\hline 6 & $\frac{\text { Ying et al. }}{(2020)}$ & Chest CT & $\begin{array}{l}777 \text { COVID- } \\
19(+) \\
708 \text { Healthy }\end{array}$ & DRE-Net & 86.0 \\
\hline 7 & $\frac{\text { Wang et al. }}{(2020 \mathrm{~b})}$ & Chest CT & $\begin{array}{l}195 \text { COVID- } \\
19(+) \\
258 \text { COVID-19(- } \\
)\end{array}$ & M-Inception & 82.9 \\
\hline 8 & $\frac{\text { Zheng et al. }}{(2020)}$ & Chest CT & $\begin{array}{l}313 \text { COVID- } \\
19(+) \\
229 \text { COVID-19(- } \\
)\end{array}$ & $\begin{array}{l}\text { UNet+3D Deep } \\
\text { Network }\end{array}$ & 90.8 \\
\hline 9 & $\underline{\mathrm{Xu} \text { et al. }(2020)}$ & Chest CT & $\begin{array}{l}219 \text { COVID-19(- } \\
\text { ) } \\
224 \text { Viral } \\
\text { pneumonia } \\
75 \text { Healthy }\end{array}$ & $\begin{array}{l}\text { ResNet + Location } \\
\text { Attention }\end{array}$ & 86.7 \\
\hline \multirow[t]{5}{*}{10} & \multirow{5}{*}{$\frac{\text { Khalid and }}{\text { Youness (2020) }}$} & \multirow{5}{*}{$\begin{array}{l}\text { Chest X-ray } \\
\text { and CT Scan }\end{array}$} & \multirow{5}{*}{$\begin{array}{l}2780 \text { bacterial } \\
\text { pneumonia } \\
1493 \text { corona- } \\
\text { virus } \\
231 \text { Covid19 } \\
1583 \text { normal }\end{array}$} & Inception_Resnet_V2 & 92.18 \\
\hline & & & & DensNet201 & 88.09 \\
\hline & & & & Resnet50 & 87.54 \\
\hline & & & & Mobilenet_V2 & 85.47 \\
\hline & & & & Inception_V3 & 88.03 \\
\hline
\end{tabular}




\begin{tabular}{|c|c|c|c|c|c|}
\hline & & & & VGG-16 & 74.84 \\
\hline & & & & VGG-19 & 72.52 \\
\hline \multirow[t]{2}{*}{11} & \multirow[t]{2}{*}{$\frac{\text { Tulin et al. }}{(2020)}$} & \multirow[t]{2}{*}{ Chest X-ray } & $\begin{array}{l}\text { 2-Class: } \\
125 \text { COVID- } \\
19(+) \\
500 \text { No-Findings }\end{array}$ & DarkCovidNet & 98.08 \\
\hline & & & $\begin{array}{l}\text { 3-Class: } \\
125 \text { COVID- } \\
19(+) \\
500 \text { Pneumonia } \\
500 \text { No-Findings }\end{array}$ & DarkCovidNet & 87.02 \\
\hline \multirow[t]{2}{*}{12} & \multirow[t]{2}{*}{$\begin{array}{l}\text { Elzeki et al. } \\
(2021)\end{array}$} & \multirow[t]{2}{*}{ Chest X-ray } & $\begin{array}{l}\text { 2-Class: } \\
221 \text { COVID- } \\
19(+) \\
234 \text { Normal }\end{array}$ & CXRVN Network & 96.7 \\
\hline & & & $\begin{array}{l}\text { 3-Class: } \\
221 \text { COVID- } \\
19(+) \\
148 \text { Pneumonia } \\
234 \text { Normal } \\
\end{array}$ & CXRVN Network & 93.07 \\
\hline 13 & $\begin{array}{l}\text { Irfan et al. } \\
(2021)\end{array}$ & $\begin{array}{l}\text { Chest X-ray } \\
\text { and Chest CT }\end{array}$ & $\begin{array}{l}3500 \text { COVID-19 } \\
\text { and } 1500 \text { healthy }\end{array}$ & $\begin{array}{l}\mathrm{CNN} \text { and Long-short } \\
\text { term memory }\end{array}$ & $99 \%$ \\
\hline \multirow[t]{4}{*}{14} & \multirow[t]{4}{*}{ Proposed Study } & \multirow[t]{4}{*}{ Chest X-ray } & \multirow{2}{*}{$\begin{array}{l}\text { 2-Class: } \\
1,300 \text { COVID- } \\
19(+) \\
1,300 \text { Normal }\end{array}$} & VGG-16 & 99.23 \\
\hline & & & & VGG-19 & 98.00 \\
\hline & & & \multirow{2}{*}{\begin{tabular}{|l|} 
3-Class: \\
1,300 COVID- \\
$19(+)$ \\
1,300 Viral \\
Pneumonia \\
1,300 Normal
\end{tabular}} & VGG-16 & 93.85 \\
\hline & & & & VGG-19 & 92.92 \\
\hline
\end{tabular}

